\title{
Biosynthetic heme of malaria parasite induces cerebral pathogenesis by regulating hemozoin formation and griseofulvin can prevent cerebral \\ malaria
}

Manjunatha Chandana ${ }^{1,2,4}$, Aditya Anand ${ }^{1,4}$, Sourav Ghosh ${ }^{1}$, Subhashree Beura ${ }^{1}$, Sarita Jena ${ }^{1}$, Amol Ratnakar Suryawanshi ${ }^{1}$, Govindarajan Padmanaban ${ }^{3}$ and Viswanathan Arun Nagaraj ${ }^{1, *}$ ${ }^{1}$ Infectious Disease Biology, Institute of Life Sciences, Bhubaneswar - 751023, Odisha, India ${ }^{2}$ School of Biotechnology, Kalinga Institute of Industrial Technology, Bhubaneswar 751024, Odisha, India

${ }^{3}$ Department of Biochemistry, Indian Institute of Science, Bangalore - 560012, Karnataka, India

${ }^{4}$ These authors contributed equally

*Corresponding author: Email: arun@ils.res.in

\begin{abstract}
Heme-biosynthetic pathway of malaria parasite is dispensable for asexual stages, but essential for sexual and liver stages. Despite having backup mechanisms to acquire hemoglobin-heme, pathway intermediates and/or enzymes from the host, asexual parasites express heme pathway enzymes and synthesize heme. Here we show heme synthesized in asexual stages promotes cerebral pathogenesis by enhancing hemozoin formation. Hemozoin is a parasite molecule associated with inflammation, aberrant host-immune responses, disease severity and cerebral pathogenesis. The heme pathway knockout parasites synthesize less hemozoin, and mice infected with knockout parasites are completely protected from cerebral malaria and death due to anaemia is delayed. Biosynthetic heme regulates food vacuole integrity and the food vacuoles from knockout parasites are compromised in $\mathrm{pH}$, lipid unsaturation and proteins, essential for hemozoin formation. Targeting parasite heme synthesis by griseofulvin
\end{abstract}


- a FDA-approved drug, prevents cerebral malaria in mice and provides a new adjunct therapeutic option for cerebral and severe malaria.

\section{INTRODUCTION}

Malaria remains a major concern of morbidity and mortality, especially with the emerging parasite resistance to artemisinin-based combination therapies (ACTs) and mosquito resistance to insecticides. According to World Health Organization (WHO), 229 million cases and 409,000 malaria deaths occurred in $2019^{1}$. Of the five Plasmodium species causing human malaria, Plasmodium falciparum (Pf) is the deadliest one responsible for more than $90 \%$ of the infections. The clinical manifestations of $P f$ malaria vary from mild (uncomplicated malaria) to severe (complicated malaria). Uncomplicated malaria is characterized by fever, headache, nausea, chills and mild anaemia. Complicated malaria is categorized by the existence of at least one criterion of disease severity that includes respiratory distress, metabolic acidosis, pulmonary edema, severe anaemia, jaundice, renal failure or neurological complications like impaired consciousness, convulsions etc. The typical outcome of severe malaria is multi-organ failure and/or cerebral malaria $(\mathrm{CM})$ of which, $\mathrm{CM}$ is the most severe neurological complication with high mortality. About one-third of the patients recovering from CM show long-term neurocognitive impairments ${ }^{2,3,4}$.

Our current understanding on $\mathrm{CM}$ comes from a few post-mortem studies of human $\mathrm{CM}$ $(\mathrm{HCM})$ and a large number of experimental CM (ECM) studies performed in mouse models $^{5,6}$. There occurs increased permeability of blood-brain barrier (BBB), brain capillary occlusions, parasitized-red blood cells (pRBCs) accumulation in brain microvasculature, and endothelial activation with dysregulated inflammation and aberrant host-immune responses $^{7,8,9,10,11,12,13,14,15}$ that lead to BBB disruption, intracerebral hemorrhages, ischemia, 
edema, increased intracranial pressure, axonal damage and demyelination, culminating in the dysfunction of central nervous system ${ }^{16,17,18,19,20,21,22,23}$. Cerebral pathology arises due to a complex interplay of molecular events triggered by various host- and parasite-derived factors. The synchronous growth of the asexual parasites in red blood cells (RBCs) and the associated schizogony result in the release of pathogen-associated molecular patterns (PAMPs) such as hemozoin (Hz), glycosylphosphatidylinositiol, parasite DNA and RNA, and dangerassociated molecular patterns (DAMPs) such as heme, uric acid, and microvesicles ${ }^{14,15}$. Hz and its precursor heme play a central role in CM pathogenesis ${ }^{14,24,25,26,27,28,29,30,31}$. The asexual stage parasites endocytose host hemoglobin $(\mathrm{Hb})$ and digest it in the food vacuole (FV). The toxic free heme released during this process is detoxified into $\mathrm{Hz}$ by heme detoxification protein (HDP) that undergoes a circuitous trafficking and abundantly present in infected RBCs than parasite $\mathrm{FVs}^{32}$. Although the rate of HDP-mediated $\mathrm{Hz}$ formation is much higher $^{32}$, autocatalytic ${ }^{33}$, histidine rich protein (HRP)-mediated ${ }^{34}$ and lipid-driven mechanisms ${ }^{35,36}$ for $\mathrm{Hz}$ formation have also been described. There is a positive correlation of $\mathrm{Hz}$ released into the circulation and phagocytosed by the circulating phagocytic cells with disease severity in children and adults ${ }^{37,38,39}$. Similarly, plasma free heme is associated with disease severity ${ }^{40,41}$. Free heme is extremely cytotoxic to endothelial cells and it can increase the expression of adhesion molecules, induce NLRP3 inflammasome and IL-1 $\beta$ secretion, and activate polymorphonuclear cells. The only treatment option for CM is parenteral administration of artemisinin derivatives or quinine with supportive therapies. However, the fatality due to $\mathrm{CM}$ remains high despite the parasite clearance ${ }^{2,4,42}$. The molecular mechanisms underlying $\mathrm{CM}$ pathogenesis need to be understood for developing adjunct therapies. 
Malaria parasite synthesizes heme de novo despite the ability of asexual stages to access host $\mathrm{Hb}_{\mathrm{b}} \mathrm{me}^{43}$. The parasite heme pathway is compartmentalized in mitochondrion, apicoplast and cytosol, and heme is eventually synthesized in the mitochondrion. Our earlier study with P. berghei $(\mathrm{Pb})$ aminolevulinate synthetase (ALAS) and ferrochelatase (FC) knockouts (KOs) generated for the first and last enzymes, demonstrated that the parasite pathway is dispensable for asexual stages, but essential for the development of sporozoites in mosquitoes and pre-erythrocytic stages in liver. Moreover, FCKO parasites can utilize host Hb-heme for their survival in blood stages ${ }^{44,45}$. Subsequent studies in $P f$ using the KO parasites generated for ALAS, FC, apicoplast-localized porphobilinogen deaminase and cytosol-localized coproporphyrinogen oxidase, confirmed these findings with the suggestion that extracellular ALA acquired through new permeability pathways may lead to parasite heme synthesis ${ }^{46,47}$. The conversion of extracellular ALA into protoporphyrin IX occurs in RBCs with the help of host enzymes, and heme is synthesized by parasite FC. Although de novo heme synthesis is non-essential and the asexual KO parasites can acquire heme/heme precursors from host RBCs and import some of the host enzymes, the parasite enzymes are expressed and heme synthesis occurs as evident from ${ }^{13} \mathrm{C} /{ }^{14} \mathrm{C}$-ALA metabolic labelling studies $^{43,44,45,46,47,48,49,50,51,52,53,54,55,56}$. We hypothesized that the parasite de novo heme pathway should have a physiological relevance in the asexual stages. Here, we demonstrate that de novo heme induces $\mathrm{CM}$ pathogenesis by regulating $\mathrm{Hz}$ formation in the asexual stages, thus offering an answer to a long-standing question. We further provide a therapeutic option on the basis that griseofulvin - a well-known FDA-approved drug capable of inhibiting parasite heme synthesis, can prevent $\mathrm{CM}$ in mice.

\section{RESULTS}

Mice infected with heme pathway KO parasites are protected from CM 
Our earlier work with $\mathrm{Pb}$ heme pathway KO parasites was carried out in outbred Swiss mice that do not develop $\mathrm{CM}^{44}$. Here, we performed our studies in $\mathrm{CM}$-susceptible C57BL/6 inbred mouse strain by injecting $10^{5}$ asexual stage parasites. Assessment of the peripheral blood parasitemia showed 2-3 days delay in the growth of $\mathrm{KO}$ parasites with respect to the WT (Fig. 1a). Importantly, about $80 \%$ of the WT-infected mice succumbed to CM within day 10 when the blood parasitemia was around $10-30 \%$. The WT-infected mice that escaped from CM died of anaemia on day 12-16 post-infection. In contrast, mice infected with ALASKO and FCKO parasites were completely protected from CM and they died because of anaemia on day 20-30 (Fig. 1b). The delay in the growth of KO parasites was associated with an early increase in the spleen weight of infected mice (Fig. 1c), suggesting a better splenic clearance. A similar delay in the growth of $\mathrm{KO}$ parasites and the mortality of KO-infected mice due to anaemia was observed in Balb/c mice that do not develop CM (Supplementary Fig. 1a,b). To rule out the possibility that $\mathrm{CM}$ protection is because of a delay in the increase in blood parasitemia, we performed growth analyses in C57BL/6 mice infected with $10^{7}$ ALASKO/FCKO parasites. While the growth of $10^{7} \mathrm{KO}$ parasites in mice was comparable with $10^{5}$ WT parasites, KO-infected mice were once again completely protected from $\mathrm{CM}$ (Fig. 1d,e). The mortality due to anaemia was delayed by 6-10 days, and KO-infected mice could sustain a higher parasitemia for a prolonged period. There were no significant differences in the reticulocyte versus mature RBC preference between $\mathrm{WT}$ and $\mathrm{KO}$ parasites in the first 9 days, the duration in which CM mortality occurred in WT-infected mice. However, KO parasites showed significantly increased reticulocyte preference and multiple infections in the reticulocytes during the later course of infections that represent anaemic phase (Fig. 1f,g). The rapid murine coma and behavioural scale (RMCBS) score of $10^{5} \mathrm{WT}$ parasite-infected mice that succumbed to $\mathrm{CM}$ was below 5 on day 7 whereas, RMCBS score of $10^{7} \mathrm{KO}$ parasite-infected mice was around 17 and 14 on day 7 and 14, respectively (Fig. 
1h). For the subsequent experiments, we initiated asexual infections by injecting $10^{5} \mathrm{WT}$ and $10^{7}$ ALASKO/FCKO parasites. These results were confirmed with another set of independent KO parasite lines wherein, ALAS and FC genes were replaced individually with GFPluciferase (Luc)-expressing cassette containing m-cherry (Fig. 2a). The successful replacement of ALAS and FC was confirmed by PCR analyses performed with DNA and RNA isolated from the respective KO parasites (Fig. 2b,c), and by examining GFP and mcherry fluorescence (Fig. 2d). For control, c/d ssurRNA locus in the WT parasite was replaced with GFP-Luc-expressing cassette containing m-cherry. In vivo bioluminescence studies showed accumulation of pRBCs in the brain of WT-infected mice, but not in the KO-infected mice (Fig. 2e) and this was confirmed by ex vivo imaging as well (Fig. 2f). The mice infected with Luc-expressing KO parasites were also completely protected from CM (Fig. 2g). These data indicated that the mice infected with heme pathway KO parasites are completely protected from CM.

\section{Absence of cerebral pathology in heme pathway KO parasite-infected mice}

To evaluate the integrity of BBB and assess vascular leakage, Evans blue extravasation analyses were carried out. While the brain collected from WT-infected mice on day $7 / 8$ stained intensely with Evans blue, the extravasation of Evans blue into the brain was barely detectable in KO-infected mice on day 7 and 14 (Fig. 3a). Quantification of Evans blue in the brain extracts confirmed this observation (Fig. 3b). Histopathological assessment of hematoxylin and eosin (H\&E)-stained brain sections of WT-infected mice on day 7 showed intracerebral hemorrhages with extravasation of erythrocytes into the perivascular space, petechial hemorrhages, thrombosed and leukocyte-packed vessels, gross demyelination and myelin pallor. No such hallmark features of CM could be detected in the brain sections of KO-infected mice (Fig. 3c). Immunohistochemical studies performed with the brain sections 
of WT-infected mice showed the extravasation of IgG in cerebral parenchyma and the presence of IgG in occluded vessels and hemorrhages, but not in the KO-infected mice (Fig. 3d). Luminal and abluminal leukocytes, and parasite-derived $\mathrm{Hz}$ could be detected in the occluded vasculature of WT-infected mice. (Fig. 3e). Immunofluorescence analyses of the brain sections using $\mathrm{Pb}$ glyceraldehyde-3-phosphate dehydrogenase (GAPDH) and mouse CD31 antibodies showed the accumulation of parasites in $\mathrm{CD}^{+} 1^{+}$vasculature and extravascular parasites in the hemorrhages of WT-infected mice, but not in the KO-infected mice (Fig. 3f). Similarly, antibodies specific for CD3 and $\beta$-amyloid precursor protein ( $\beta$ APP) showed the accumulation of $\mathrm{CD}^{+} \mathrm{T}$ cells in the cerebral vasculature (Fig. 3g) and axonal injury in the brain sections of WT-infected mice, but not in the KO-infected mice (Fig. 3h). These data suggested the absence of CM-associated brain lesions in mice infected with heme pathway KO parasites.

\section{Levels of inflammatory parameters in heme pathway KO parasite-infected mice}

Multiplex assays performed for mice infected with $\mathrm{KO}$ parasites on day 8 showed a significant decrease in the plasma levels of proinflammatory cytokines and chemokines - IL6, TNF $\alpha$, IFN $\gamma$, G-CSF, CCL3 (MIP-1 $\alpha$ ) and CCL5 (RANTES). There was also a significant increase in anti-inflammatory cytokines - IL-4, IL-10 and IL-13 (Fig. 4a). Quantitative RTPCR analyses examining the expression of cytokines, chemokines, chemokine receptors and other key mediators of cerebral pathogenesis in the brain samples of KO-infected mice showed a substantial reduction of more than 1.5 fold in the transcript levels of TNF $\alpha$, IFN $\gamma$, CXCL9, CXCL10, CCL2 (MCP-1), CCL5, CCL19, perforin, granzyme B, ICAM-1, pselectin and HO-1. In particular, the decrease in IFN $\gamma$, CXCL10, CCL2, CCL5, granzyme B, ICAM-1 and HO-1 in FCKO was greater than 4-fold (Fig. 4b). Flow cytometry analyses of the leukocytes isolated from the brain samples of KO-infected mice showed significant 
reduction in $\mathrm{CD}^{+} \mathrm{CD}^{+}$and $\mathrm{CD}^{+} \mathrm{CD}^{+}$double positive cells, and $\mathrm{CD}^{+} \mathrm{CD}^{+} \mathrm{CD}^{+} 9^{+}, \mathrm{CD}^{+}$ $\mathrm{CD}^{+} \mathrm{CXCR}^{+}, \mathrm{CD}^{+} \mathrm{CD}^{+}$perforin $^{+}, \mathrm{CD}^{+} \mathrm{CD}^{+}$granzyme $\mathrm{B}^{+}, \mathrm{CD}^{+} \mathrm{CD}^{+} \mathrm{TNF}^{+}$and $\mathrm{CD}^{+} \mathrm{CD}^{+} \mathrm{IFN}^{+}$triple positive cells (Fig. 4c and Supplementary Fig. 2). Western analyses for the brain homogenates of KO-infected mice showed reduction in phospho-NLRP3, phospho-NF- $\kappa$ B, cleaved caspase-1 and IL-1 $\beta$ (Fig. 4d). These data indicated an overall decrease in systemic and neuronal inflammation, and $\mathrm{T}$ cell infiltration in the brain milieu, suggesting that the cerebral pathology is impeded in mice infected with heme pathway KO parasites.

\section{Decreased $\mathrm{Hz}$ formation in heme pathway KO parasites}

A substantial decrease for $\mathrm{Hz}$ formation in the asexual stages of $\mathrm{KO}$ parasites was observed in the Giemsa-stained peripheral blood smears (Fig. 5a). This was more prominent in the asexual stages than gametocytes (Fig. 5b) and could be readily detected in almost $60-70 \%$ of the pRBCs containing asexual stages. These findings were verified by examining $\mathrm{Hz}$ content in paraformaldehyde-fixed pRBCs (Fig. 5c) and Hz dynamics in live pRBCs (Supplementary Movie 1, Supplementary Movie 2 and Supplementary Movie 3). To confirm, total Hz content of the parasites normalized with respect to the protein was examined and it was only $20-25 \%$ of the WT (Fig. 5d). During Hz formation in the FV, heme can leach into the parasite cytosol. Free heme in the parasite lysates of the $\mathrm{KO}$ parasites prepared by hypotonic lysis showed around 55\% decrease when compared with WT (Fig. 5e). In addition, there was a significant decrease of around 55-60\% in the plasma free heme and heme/hemopexin ratio of the KOinfected mice (Fig. 5f,g). However, there were no significant differences between the plasma hemopexin levels of WT- and KO-infected mice (Fig. 5h), and there was only a marginal 15$20 \%$ decrease in the plasma $\mathrm{Hb}$ levels that was statistically significant in case of FCKO, but not in ALASKO (Fig. 5i). The quantification of $\mathrm{Hz}$ load in the spleen and liver showed 40- 
$50 \%$ decrease in the KO-infected mice (Fig. 5j,k). A similar decrease in the Hz content and free heme was observed for the KO parasites isolated from Balb/c mice (Supplementary Fig. 3a,b). These results suggested an overall decrease in the Hz synthesis of KO parasites.

Heme and $\mathrm{Hz}$ are associated with the function of antimalarial drugs - artemisinin and chloroquine $^{57,58}$. Therefore, we were interested in examining the effect of $\alpha, \beta$-arteether (artemisinin derivative) and chloroquine on FCKO-infected C57BL/6 mice in vivo. For $\alpha, \beta$ arteether treatment, a single intramuscular dose of $1 \mathrm{mg} / \mathrm{mouse}$ or $0.4 \mathrm{mg} / \mathrm{mouse}$ was administered on day 5 post-infection when the blood parasitemia was around $5 \%$. For chloroquine treatment, a daily dose of $25 \mathrm{mg} / \mathrm{kg}$ or $10 \mathrm{mg} / \mathrm{kg}$ was administered intraperitoneally for five consecutive days from day 5 post-infection. While $1 \mathrm{mg}$ of $\alpha, \beta$ arteether could completely clear the WT and FCKO parasites, 50\% recrudescence was observed for $0.4 \mathrm{mg}$ dosage in the WT- and FCKO-infected mice. The day of recrudescence was comparable between WT and FCKO parasites and the parasites were detectable in peripheral smears prepared on day 12 post-infection. However, the growth of FCKO parasites in mice showing recrudescence was delayed in the subsequent days and this was reflected in the mortality (Fig. 6a,b). The assessment of parasite load at the time of recrudescence on day 12 using $P b G A P D H$ primers showed that $C_{t}$ values normalized against mouse GAPDH for FCKO-infected mice were approximately 1.5 cycles more than WT-infected mice (Supplementary Fig. 4a-d), suggesting 3-fold less parasite load in FCKO-infected mice. In case of chloroquine, recrudescence was observed for WT and FCKO parasites with both the doses that were tested and importantly, FCKO parasites appeared 2-4 days earlier than WT (Fig. 6c,d). This was also confirmed by performing qPCR analysis on day 13 for $25 \mathrm{mg} / \mathrm{kg}$ and day 10 for $10 \mathrm{mg} / \mathrm{kg}$ doses. In both the cases, $C_{t}$ values obtained for the parasite load in FCKO-infected mice were approximately 4 cycles lower than WT-infected mice, suggesting 
16-fold higher parasite load in FCKO-infected mice (Supplementary Fig. 4e-h). Further, one out of four and two out of four WT-infected mice died of CM in $25 \mathrm{mg} / \mathrm{kg}$ and $10 \mathrm{mg} / \mathrm{kg}$ treatment, respectively. Despite showing an early recrudescence, all the FCKO-infected mice died of anaemia and their mortality was delayed in comparison to WT-infected mice (Fig. 6d). These results indicated that chloroquine sensitivity is compromised in FCKO parasites while $\alpha, \beta$-arteether sensitivity remains almost unaltered in vivo in mice.

\section{De novo heme is essential for the functional integrity of FV}

To understand the molecular mechanisms underlying decreased $\mathrm{Hz}$ synthesis, we assessed $\mathrm{pH}$, lipids and protein content of the FVs in FCKO parasites and compared with WT parasites. Live fluorescence imaging of pRBCs incubated with acidophilic dye LysoTracker Deep Red indicated less fluorescence in the FCKO parasites (Fig. 7a), suggesting that the pH of FCKO FV is compromised. The quantification of fluorescence signal intensities showed $\sim 50 \%$ decrease (Fig. 7b). We examined the phospholipid content and could not find significant differences for in vitro ${ }^{32} \mathrm{P}$-orthophosphoric acid radiolabelling of major phospholipids - phosphatidylcholine (PC), phosphatidylinositol (PI) and phosphatidylethanolamine (PE), in the total parasites and FVs of FCKO parasites (Fig. 7c,d and Supplementary Fig. 5). Similarly, the assessment of neutral lipid content by live fluorescence imaging of pRBCs stained with BODIPY 493/503 and Nile Red did not show significant differences (Fig. 7e-h). Lipids associated with parasite $\mathrm{Hz}$ are reported to be abundant in monohydroxy derivatives of polyenoic fatty acids, and unsaturated glycerophospholipids induce rapid and efficient $\mathrm{Hz}$ formation in hematophagous insect Rhodnius prolixus ${ }^{59,60}$. GC-MS analysis of fatty acid methyl esters (FAMEs) prepared from the FVs of $P b$ parasites indicated the presence of oleic acid (OA) as a major unsaturated fatty acid followed by arachidonic acid along with other saturated fatty acids, fatty acyl alcohols 
and derivatives, alkanes, etc. (Supplementary Fig. 6a and Supplementary Table 1). Malaria parasite scavenges stearic acid (SA) from plasma and converts it into OA by ER-localized 49-desaturase (stearoyl-CoA 9-desaturase). The cis double bond formation in OA requires heme since it utilizes the electrons transferred by cytochrome b5 from NADH cytochrome b5 reductase ${ }^{61,62}$. In vitro ${ }^{14} \mathrm{C}-\mathrm{SA}$ radiolabelling and the separation of unsaturated FAMEs prepared from FCKO total parasites and FVs showed almost $80-90 \%$ decrease in the levels of OA methyl ester (OAME) in comparison to WT (Fig. 8a-c), with no significant differences in the signal intensities of SA methyl ester (SAME). The identity of the slower migrating polyunsaturated FAME (PU-FAME) that also showed a significant decrease is yet to be established and there are no clear-cut evidences for the presence of other desaturases in the parasite. There was a significant decrease in the radiolabelling of FV, but not in the total parasites (Supplementary Fig. 6b).

FV proteomics is challenging due to the presence of large amounts of host $\mathrm{Hb}, \mathrm{Hb}$ degradation peptides and indigenous proteases. There is only one report on $P f \mathrm{FV}$ proteomics based on LC-MS/MS analysis of proteins excised from SDS-PAGE gel that identified 116 proteins excluding elongation factors and ribosomal proteins ${ }^{63}$. Our repeated attempts to perform differential proteomics for WT and FCKO FVs by Isobaric tags for relative and absolute quantitation (iTRAQ) were unsuccessful because of the interference from host $\mathrm{Hb}$. LC-MS/MS of in-solution trypsin digested FV protein extracts solubilized with $6 \mathrm{M}$ urea was successful. The quality of FV preparations could be assessed by the presence of signature FV proteins such as plasmepsin IV, berghepain, aminopeptidases, subunits of vacuolar-type $\mathrm{H}^{+}$ATPase (V-type $\mathrm{H}^{+}$ATPase), together with parasitophorous vacuolar (PV) proteins including exported protein 1 (Exp1), Exp2, early transcribed membrane protein, PV1, PV5 (lipocalin) etc., and Rab GTPases associated with cytostome-FV trafficking. A total number 
of 251 and 201 proteins could be identified for WT and FCKO FVs, respectively, and 175 proteins were common between them suggesting an overall consistency in the preparations (Fig. 8d). Around $68 \%$ of the proteins identified in the total Pf FV proteome were present in our preparations. Remarkably, in agreement with the decreased uptake of LysoTracker Deep Red in FCKO pRBCs, none of the subunits of V-type $\mathrm{H}^{+}$ATPase - a proton pump maintaining the acidic $\mathrm{pH}$ of $\mathrm{FV}^{64}$ could be detected in FCKO FVs indicating the less abundancy of these proteins. In WT FV preparations, A, B and $\mathrm{G}$ subunits of V-type $\mathrm{H}^{+} \mathrm{ATPase}$ could be detected. While plasmepsin $\mathrm{IV}$, the only $\mathrm{Pb}$ aspartic protease involved in $\mathrm{Hb}$ degradation could be detected in WT and FCKO FVs, berghepain-2 - a cysteine protease involved in $\mathrm{Hb}$ degradation $^{65,66}$ could not be detected in FCKO FVs (Fig. 8e, Supplementary Table 2 and Supplementary Table 3). The rest of the proteins unique for WT and FCKO FVs include proteasome subunits, ribosomal proteins, metabolic enzymes etc., (Supplementary Table 2 and Supplementary Table 3) that are not related to $\mathrm{Hz}$ formation and known to be present in FV preparations ${ }^{63}$. All these evidences suggested that the functional integrity of FCKO FVs is compromised in terms of $\mathrm{pH}$, lipid unsaturation and proteins that in turn can collectively lead to a decreased $\mathrm{Hz}$ formation.

\section{Griseofulvin treatment protects mice from ECM}

Griseofulvin, isolated from Penicillium griseofulvum, is a FDA-approved antifungal drug used to cure tinea infections. It interacts with fungal microtubules and disrupts spindle assembly leading to mitotic arrest. In humans, griseofulvin dosage is given to the extent of $1000 \mathrm{mg} /$ day in adults and $10 \mathrm{mg} / \mathrm{kg} /$ day in children for several weeks ${ }^{67,68,69}$. It can also inhibit FC by generating N-methyl protoporphyrin IX (NMPP) through the action of cytochrome P450 enzymes ${ }^{70}$. Therefore, we evaluated the potential of griseofulvin in preventing $\mathrm{CM}$ by treating WT-infected $\mathrm{C} 57 \mathrm{BL} / 6$ mice from day 4 when the blood 
parasitemia was around 2\%. A single dose of $2 \mathrm{mg}$ /day (comparable with the dosage of humans) administered from day 4 and continued until day 8 showed the best protection. While $\sim 80 \%$ of the control mice succumbed to CM within day 10 , more than $80 \%$ of the treated mice were protected from CM. Similar protection was also observed for the mice treated with $1 \mathrm{mg}$ dose, twice a day from day 4 to day 8 (Fig. 9a). There was also a significant delay in the mortality that occurred due to anaemia. While the mortality in CMescaped control mice occurred within day 17 , more than $80 \%$ of the treated mice could survive beyond day 20 with almost $50 \%$ of them surviving even beyond day 24 . Interestingly, the growth curves of WT parasites in treated and untreated mice were very much comparable (Fig. 9b). We analyzed heme synthesis in griseofulvin-treated WT parasites by incubating the in vivo-treated $\mathrm{pRBCs}$ in vitro with ${ }^{14} \mathrm{C}$-ALA for $9 \mathrm{~h}$ and there was around $60 \%$ decrease in the ${ }^{14} \mathrm{C}$-labelling of free heme (Fig. 9c). Further, griseofulvin-treated mice showed less Evans blue extravasation in the brain (Fig. 9d), with the absence of intracerebral hemorrhages (Fig. 9e), lack of accumulation of parasites and $\mathrm{CD}^{+} \mathrm{T}$ cells in the cerebral vasculature (Fig. 9f,g) and undetectable axonal injury (Fig. 9h). Giemsa-stained peripheral smears and paraformaldehyde-fixed pRBCs showed less $\mathrm{Hz}$ content (Fig. 9i,j). As observed for KO parasites, the total $\mathrm{Hz}$ content in the griseofulvin-treated parasites was around $50-60 \%$ less when compared with untreated parasites (Fig. 9k) and there was close to $60 \%$ decrease in the free heme levels (Fig. 91). Further, the plasma levels of heme, hemopexin and $\mathrm{Hb}$ together with heme/hemopexin ratio of the griseofulvin-treated WT-infected mice were comparable with the KO-infected mice (Fig. 9m-p). These results indicated the potential of griseofulvin in preventing $\mathrm{CM}$ through the inhibition of parasite heme synthesis.

\section{DISCUSSION}


Parasite heme pathway has remained enigmatic for more than two decades and the dichotomy between de novo heme and host-heme/porphyrin acquisition pathways in the asexual stages is obscure. Here, we provide an explanation as to why asexual parasite expresses "dispensable" heme pathway enzymes and synthesizes heme despite acquiring host heme, by demonstrating that de novo heme pathway is associated with disease virulence and it induces $\mathrm{CM}$ pathogenesis by promoting $\mathrm{Hz}$ formation. $\mathrm{Hz}$ is a key malarial PAMP associated with aberrant inflammatory responses, endothelial activation promoting $\mathrm{pRBC}$ sequestration, $\mathrm{T}$ cell infiltration and neuronal apoptosis. Upon phagocytosis, $\mathrm{Hz}$ can induce the production of pro-inflammatory cytokines and chemokines in neutrophils, monocytes, macrophages and DCs, leading to the increased expression of ICAM-1 in endothelial cells and enhanced sequestration of pRBCs. Hz can also trigger IL-1 $\beta$ production by activating NLRP3 inflammasome, and augment endothelial cell damage and loss of BBB integrity by inducing proinflammatory responses in endothelial cells and activating coagulation and complement pathways $^{14,25}$. The KO parasites synthesize less $\mathrm{Hz}$ and mice infected with $\mathrm{KO}$ parasites are completely devoid of cerebral complications. The plasma levels of IL-6, TNF $\alpha$, IFN $\gamma$, GCSF, CCL3 and CCL5 are significantly decreased in KO-infected mice. These cytokines and chemokines are known to be highly elevated in the serum samples of cerebral and severe malaria patients ${ }^{25,71,72}$. There is also a concomitant increase in anti-inflammatory cytokines such as IL-4, IL-10 and IL-13 in the KO-infected mice indicating an over-all decrease in the systemic inflammation.

The transcript levels of TNF $\alpha$, IFN $\gamma$, CXCL9, CXCL10, CCL2, CCL5 and CCL19 are low in the brain samples of KO-infected mice. CXCL9 and CXCL10 are highly induced in the brain of ECM mice and elevated levels of CXCL10 in plasma and cerebrospinal fluid predict fatal CM in humans ${ }^{73}$. CCL2, CCL5 and CCL19 associated with leukocyte migration are known to 
be induced by $\mathrm{Hz}$ in monocyte-derived DCs, macrophages and neutrophils ${ }^{14,25,26,74}$. Similar decrease was also observed in the transcript levels of ICAM-1, p-selectin, perforin, and granzyme B, the key adhesion and cytotoxic effector molecules. Low transcript levels of HO1 in KO-infected mice are due to less inflammation and decreased extracellular heme - the two potent HO-1 inducers ${ }^{29,30}$. Further, there is a decrease in the infiltration of $\mathrm{CD}^{+} \mathrm{T}$ cells expressing early activation marker CD69, and proinflammatory and cytotoxic effector molecules such as $\mathrm{TNF} \alpha^{+}, \mathrm{IFN} \gamma^{+}, \mathrm{CXCR}^{+}$, perforin and granzyme $\mathrm{B}$ that leads to endothelial leakage, $\mathrm{BBB}$ disruption and neuronal damage in $\mathrm{CM}$. There is also a reduction in phosphoNF- $\mathrm{KB}$ and NLRP3 inflammasome formation with decreased caspase-1 activation and less production of IL-1 $\beta$ - a key mediator of inflammation, infiltration of immune cells and neuronal apoptosis.

The decrease in $\mathrm{Hz}$ synthesis is reflected in the reduced $\mathrm{Hz}$ load in the organs, and decreased free heme levels in the parasites and plasma samples of KO-infected mice. It is suggested that oxidized heme derived from host $\mathrm{Hb}$ release during schizont rupture contributes to free plasma heme responsible for severe malaria and CM pathogenesis ${ }^{29,30,31}$. Interestingly, plasma levels of $\mathrm{Hb}$ and heme scavenging proteins like hemopexin in $\mathrm{KO}$-infected mice are comparable with WT-infected mice indicating that the decreased plasma heme levels are due to changes in parasite heme/Hz levels. While less $\mathrm{Hz}$ formation can be directly associated with reduced labile/free heme levels in KO parasites, the decrease in plasma free heme needs further investigations. Despite thriving in a heme-rich milieu, parasite maintains cytosolic free heme concentrations of $\sim 1.6 \mu \mathrm{M}$, comparable with $\sim 0.4-0.6 \mu \mathrm{M}$ concentrations seen in mammalian cells ${ }^{75}$. It is possible that the parasite could have evolved with protein/transporter-based mechanisms to dispose excess free heme from the cytosol and this could be the reason for reduced plasma free heme levels in KO-infected mice. For example, HRP 
capable of binding heme is secreted by the parasite. Altogether, our findings suggest that de novo heme in the blood stages can influence the levels of $\mathrm{Hz}$ and free heme - a key PAMP and DAMP associated with malaria pathogenesis. Chloroquine acts predominantly by inhibiting host $\mathrm{Hb}$-heme polymerization into $\mathrm{Hz}$ and it has been suggested that activation of artemisinin requires heme $\mathrm{e}^{57,58}$. In agreement with decreased $\mathrm{Hz}$ formation, the sensitivity of FCKO parasites to chloroquine is compromised. However, the in vivo sensitivity to $\alpha, \beta$ arteether remains unaltered and our results indicate that albeit a $\sim 50 \%$ decrease, the hostderived labile heme present in the cytosol of FCKO parasites is adequate to activate $\alpha, \beta$ arteether and the role of de novo heme is confined to cerebral pathogenesis.

The host $\mathrm{Hb}$ degradation in asexual stage parasites can release as much as $\sim 15 \mathrm{mM}$ heme ${ }^{75}$ and therefore, utilization of host heme cannot account for almost $75 \%$ decrease in the $\mathrm{Hz}$ synthesis of KO parasites. Here, we provide evidence for the unexpected role of de novo heme in influencing the detoxification of $\mathrm{Hb}$-heme into $\mathrm{Hz}$ by regulating the FV integrity in asexual stages (Fig. 10). FVs of FCKO parasites are compromised in terms of pH, lipid unsaturation and proteins associated with $\mathrm{Hz}$ formation. It is known that acidic $\mathrm{pH}$ of $\mathrm{FV}$ is critical for $\mathrm{Hb}$ digestion by proteases and subsequent $\mathrm{Hz}$ formation. The decrease in LysoTracker Deep Red uptake indicate that the FCKO FVs are less acidic. Our results also indicate the less abundancy of V-type $\mathrm{H}^{+}$ATPase subunits and berghepain-2 in FCKO FVs. V-type $\mathrm{H}^{+}$ATPase is a proton pump mainly responsible for maintaining the acidic $\mathrm{pH}$ of FV and targeting V-type $\mathrm{H}^{+}$ATPase activity with concanamycin A or bafilomycin A1 can lead to the alkalinisation of $\mathrm{FV}$ and inhibition of parasite growth ${ }^{64} . \mathrm{Pb}$ has two isoforms of berghepain - berghepain-1 and -2 of which, berghepain-1 is associated with hepatic merozoite invasion and erythrocyte tropism, and berghepain- 2 seems to be involved in $\mathrm{Hb}^{\text {digestion }}{ }^{65,66}$. Importantly, we show drastic reduction in OA synthesis of FCKO parasites with not much 
change in phospholipids or neutral lipids, suggesting an alteration in the degree of lipid unsaturation that can affect $\mathrm{Hz}$ formation. OA synthesis in malaria parasite is catalysed by a heme-dependent, ER-localized, $\Delta 9$-desaturase and it does not occur in uninfected RBCs ${ }^{61,62}$. Our results suggest the specific role of de novo heme in OA synthesis that cannot be compensated by host $\mathrm{Hb}$-heme. The levels of unsaturated fatty acids are also shown to stimulate the function of V-type $\mathrm{H}^{+}$ATPase in plants ${ }^{76}$, and the decrease in OA synthesis and lipid unsaturation can affect other cellular processes such as membrane homeostasis, protein/lipid trafficking, protein folding, cell signalling etc ${ }^{77}$. Although host Hb-heme acquisition starts from late ring stages, it is evident that FV integrity and maturation depend on de novo heme. Our results provide new functional insights on de novo heme that could be a miniscule in comparison with massive amounts of heme derived from host $\mathrm{Hb}$. Intriguingly, we show it is the de novo heme that influences the detoxification of host heme into $\mathrm{Hz}$ and disease pathogenesis. Despite the ability of blood stage parasites to manage host Hb-heme for survival, lack of de novo heme synthesis renders them less virulent. It would be interesting to examine whether (i) de novo heme regulates other metabolic pathways or organellar functions and influences the trafficking mechanisms associated with host-heme or $\mathrm{Hb}$ uptake and (ii) compartmentalization of de novo and $\mathrm{Hb}$-heme make them functionally different. Further, the accessibility of $\mathrm{Hb}$-heme to various organelles and its incorporation in hemoproteins may vary. Altogether, our findings suggest the importance of "dispensable" de novo heme pathway in the asexual stages despite its non-essentiality for parasite growth.

Finally, we show that de novo heme in the blood stages can serve as a target for malaria pathogenesis. Griseofulvin - a FDA-approved antifungal drug prevents $\mathrm{CM}$ in mice and delays death due to anaemia by inhibiting parasite heme synthesis. The ability of griseofulvin to prevent disease pathogenesis is observed despite the absence of any antimalarial treatment 
and griseofulvin treatment does not affect the parasite growth suggesting that $\mathrm{CM}$ protection is not because of the inhibition of parasite mitosis. This is in concurrence with a clinical trial conducted in malaria-infected humans where, griseofulvin treatment did not affect in vivo parasite growth although NMPP formation occurred within the parasite. The participants were rescued early with ACT and therefore, no observations were made on disease severity ${ }^{78}$. The fatality rates in malaria do not correlate with parasite clearance and therefore, targeting parasite virulence becomes important. Further, the malaria deaths that occur despite treating the infected individuals with ACTs and the decreasing efficacy of ACTs that lead to delayed parasite clearance underscore the need of an adjunct therapy in the initial stages of ACT treatment. Our study identifies a unique approach of targeting hemozoin through de novo heme to mitigate parasite virulence. Since de novo heme pathway is dispensable for asexual stages, there is a possibility of less selection pressure to result in resistance development. Repurposing of griseofulvin can serve as an excellent adjunct to ACTs for CM and severe malaria that needs to be evaluated in clinical trials.

\section{FUNDING}

This study is supported by Centre of Excellence and Innovation in Biotechnology (CEIB) grant (BT/PR13760/COE/34/42/2015), Department of Biotechnology, New Delhi, (G.P. and V.A.N.), and intramural support from Institute of Life Sciences ILS/16-19 (V.A.N.), Bhubaneswar. G.P. is a NASI-Platinum Jubilee Senior Scientist.

\section{ACKNOWLEDGEMENTS}

Thanks are due to Dr. Gulam Hussain Syed and Dr. Soumen Chakraborty for helping us with lipid labelling and transfection studies. We gratefully acknowledge the support rendered by Professor Balasubramanian Gopal, and mass spectrometry facilities at Molecular Biophysics 
Unit, Indian Institute of Science, Bangalore; Advanced Technology Platform Centre, Regional Centre for Biotechnology; and Kalinga Institute of Industrial Technology Technology Business Incubator. We acknowledge the support rendered by Mr. R. Rajendra Reddy, Institute of Life Sciences Central Proteomics Facility, and Mr. Paritosh Nath, Institute of Life Sciences Flow Cytometry Facility. We thank Malaria Research and Reference Reagent Resource Center (MR4), ATCC Manassas Virginia, for providing us with $\mathrm{Pb}$ ANKA (MRA-311) deposited by Thomas F. McCutchan, and addgene for providing us with GOMOGFP-LUC plasmid (\#60976) deposited by Olivier Silvie.

\section{AUTHOR CONTRIBUTIONS}

M.C., A.A., S.G., S.B. S.J. A.R.S. and V.A.N. performed the experiments. G.P. and V.A.N. conceived and designed the study. M.C., A.A., S.G., A.R.S., G.P. and V.A.N. analysed the data. M.C., A.A., S.G., G.P. and V.A.N. wrote the manuscript.

\section{COMPETING INTERESTS}

The authors declare no competing financial interests.

\section{MATERIALS AND METHODS}

\section{Routine propagation of $P$. berghei in mice and CM experiments}

P. berghei ANKA WT and KO parasites were propagated in $\mathrm{C} 57 \mathrm{BL} / 6$ male mice of 7-8 weeks old. Peripheral blood parasitemia was monitored by performing light microscopy for Giemsa-stained thin blood smears prepared from tail vein blood. When the blood parasitemia was around $10 \%, 10^{5} \mathrm{P}$. berghei ANKA WT or $10^{5} / 10^{7}$ ALAS/FC KO parasites were collected and injected intraperitoneally in 7-8 weeks old C57BL/6 male naïve mice to initiate CM experiments. CM phenotype was also confirmed with C57BL/6 female mice. For 
experiments carried out with Balb/c mice, 7-8 weeks old female mice were used. All the studies involving mice were carried out with the approval of Institutional Animal Ethics Committee (ILS/IAEC-57-AH/JAN-16) according to the national guidelines framed by "The Committee for the Purpose of Control and Supervision of Experiments on Animals (CPCSEA)". Growth curve analysis was carried out by monitoring the blood parasitemia. The development and progression of ECM were monitored by examining RMCBS for neurological symptoms as described earlier ${ }^{79}$. To assess BBB integrity, Evans blue uptake assays were carried out by injecting $200 \mu \mathrm{l}$ of $2 \%$ Evans blue in PBS intravenously and examining the extravasation of dye after one hour in the brain of the infected mice that were transcardially perfused with PBS. The extent of BBB damage was quantified by incubating the brain samples in formamide at $37^{\circ} \mathrm{C}$ for $48 \mathrm{~h}$, extracting the Evans blue and measuring the absorbance at $620 \mathrm{~nm}^{29}$.

\section{Histological and immunofluorescence analyses of cerebral pathology in the brain of infected mice}

For H\&E staining to assess vascular blockage, hemorrhages and demyelination, brain samples were fixed with formalin for 72 hours at room temperature. After dehydrating with ethanol and treating with xylene, paraffin embedded blocks were made and sections of $7 \mu \mathrm{m}$ thickness were prepared using Leica RM2125RT rotary microtome. The sections were then processed and stained with $\mathrm{H} \& \mathrm{E}$ using standard protocols. Immunohistochemical analysis of IgG extravasation in the brain sections was carried out as described ${ }^{13}$. In brief, brain sections of $30 \mu \mathrm{m}$ thickness were antigen retrieved by treating them at $95^{\circ} \mathrm{C}$ for $30 \mathrm{~min}$ in sodium citrate buffer $\mathrm{pH} 6.0$, followed by blocking with $3 \% \mathrm{H}_{2} \mathrm{O}_{2}$ at room temperature for 30 min to prevent endogenous peroxidase activity. The sections were then incubated with HRPconjugated goat anti-mouse IgG (Abcam, ab97023) at 1:250 dilution in PBS containing 0.3\% 
Triton X-100 and 0.1\% BSA, followed by developing with diaminobenzidine tertrahydrochloride (Vector Labs, SK-4100) and counterstaining with hematoxilin (HiMedia, S058). Immunoflourescence analysis of brain sections for parasite sequestration was carried out as described ${ }^{13}$ by fixing the brain samples in $4 \%$ paraformaldehyde in PBS containing $20 \%$ sucrose for $24 \mathrm{~h}$ at $4^{\circ} \mathrm{C}$ and cryoprotecting them for $48 \mathrm{~h}$ in PBS containing $20 \%$ sucrose. Coronal sections of $30 \mu \mathrm{m}$ thickness were prepared using Leica CM1850 cryostat microtome and antigen retrieval was carried out by treating them at $95^{\circ} \mathrm{C}$ for $30 \mathrm{~min}$ in sodium citrate buffer $\mathrm{pH}$ 9.0. After blocking with 1\% BSA, the sections were incubated with anti-CD31 mouse monoclonal antibody (1:200 dilution) conjugated with Alexa Fluor 594 (SantaCruz, sc-376764) and anti-PbGAPDH rabbit polyclonal serum (1:100 dilution) or antimouse CD3 rat monoclonal antibody (Invitrogen, 14-0032-82; 1;100 dilution) for $16 \mathrm{~h}$ at $4^{\circ} \mathrm{C}$. The sections were then treated with FITC-conjugated donkey anti-rabbit IgG (SantaCruz, sc2090) or FITC-conjugated goat anti-rat IgG (SantaCruz, sc-2011; 1:200 dilution), followed by 4',6-diamidino-2-phenylindole (DAPI) staining. Anti-mouse $\beta$-APP rabbit polyclonal antibody (Thermo Fisher Scientific, 51-2700) was used in 1:200 dilution. All the images were captured using Olympus IX83 microscope with DP73 high-performance camera.

\section{Heme, Hz, hemoglobin and hemopexin estimations}

Free heme levels in the plasma samples of WT- and KO-infected mice were quantified using Hemin colorimetric assay kit (BioVision, K672) as per the manufacturer's protocol. The assay is specific for free heme and it utilizes peroxidase activity of hemin to facilitate the conversion of a colorless probe to a strongly colored compound with absorbance at $570 \mathrm{~nm}$. The quantification of free heme in the parasite lysates was carried out by resuspending the parasite pellets in 5 volumes of hypotonic lysis buffer containing $5 \mathrm{mM}$ Tris $\mathrm{pH} 7.5$ with protease inhibitors and incubating them in ice for $30 \mathrm{~min}$. The lysates were then centrifuged 
at $20,000 \mathrm{~g}$ for $20 \mathrm{~min}, 4^{\circ} \mathrm{C}$, and the supernatants obtained were used for free heme estimation as mentioned above for the plasma samples. The protein content of the hypotonic lysates was measured by Micro BCA protein assay kit (Thermo Scientific, 23235) and the free heme levels were expressed per mg of protein. The Hz content of the WT and KO parasite pellets was estimated as described earlier ${ }^{80}$. In brief, the parasite pellet was resuspended in $1 \mathrm{ml}$ of $100 \mathrm{mM}$ sodium acetate buffer, $\mathrm{pH} 5.0$ and left at $37^{\circ} \mathrm{C}$ for overnight, followed by centrifugation at $10,000 \mathrm{~g}$ for $5 \mathrm{~min}$. The resultant pellet was resuspended in $100 \mathrm{mM}$ Tris buffer $\mathrm{pH} 8.0$ containing $2.5 \%$ SDS and incubated at $37^{\circ} \mathrm{C}$ for $30 \mathrm{~min}$, followed by centrifugation at $10,000 \mathrm{~g}$ for $5 \mathrm{~min}$. The pellet obtained was washed once with $100 \mathrm{mM}$ sodium bicarbonate $\mathrm{pH} 9.2$ and then with distilled water. The final $\mathrm{Hz}$ pellet was dissolved in $100 \mathrm{mM} \mathrm{NaOH}$ containing 2.5\% SDS, and the absorbance was measured at $405 \mathrm{~nm}$. The supernatants of sodium acetate and Tris SDS steps were collected to estimate the protein content and heme content of the $\mathrm{Hz}$ was expressed per mg of total protein. To estimate the $\mathrm{Hz}$ content in the spleen and liver of the WT- and KO-infected mice, $50 \mathrm{mg}$ tissue of the respective organs was homogenized in $50 \mathrm{mM}$ Tris $\mathrm{pH} 8.0$ containing $50 \mathrm{mM} \mathrm{NaCl}, 5 \mathrm{mM}$ $\mathrm{CaCl}_{2}$ and $1 \%$ Triton $\mathrm{X}-100$, and incubated for $12 \mathrm{~h}$ at $37^{\circ} \mathrm{C}$ in the presence of proteinase $\mathrm{K}$. The lysates were sonicated and centrifuged at 15,000 $\mathrm{g}$ for $30 \mathrm{~min}$. The pellets obtained were resuspended with $100 \mathrm{mM}$ sodium bicarbonate containing 2\% SDS and sonicated, followed by centrifugation at $15,000 \mathrm{~g}$ for $15 \mathrm{~min}$. After repeating this step thrice, the pellets were solubilized in $100 \mathrm{mM} \mathrm{NaOH}$ containing 2\% SDS and $3 \mathrm{mM}$ EDTA, and the absorbance was measured at $405 \mathrm{~nm}^{81}$. In parallel, the parasite load in the organs was examined by quantitative PCR (qPCR) analysis carried out with Pb18SrRNA primers for the total RNA isolated from $30 \mathrm{mg}$ tissue of the organs using RNeasy Plus Mini Kit (Qiagen, 74134). After normalizing with respect to the parasite load, the total heme content of the $\mathrm{Hz}$ isolated was expressed per mg weight of the organ. Hemoglobin and hemopexin levels in the plasma 
samples of WT- and KO-infected mice were measured by Mouse Hemoglobin (Abcam, ab157715) and Mouse Hemopexin (Abcam, ab157716) ELISA kits as per the manufacturer's protocols.

\section{In vivo bioluminescence imaging}

In vivo bioluminescence imaging for mice infected with $\mathrm{WT}$ and $\mathrm{KO}$ parasite lines expressing luciferase was carried out as described earlier ${ }^{82}$. Luc-expressing ALASKO and FCKO parasites were generated by transfecting WT parasites with GOMO-GFP-Luc plasmid containing GFP-Luc-expressing cassette with m-cherry flanked on either side by 5'- and 3'UTR regions of the respective genes. The primers utilized were similar to the earlier ones ${ }^{44}$ except that the restriction sites used for forward and reverse primers were SacII and NotI for 5' UTRs and XhoI and KpnI for 3' UTRs, respectively. For Luc-expressing WT parasites, the following sets of forward and reverse primers were used to amplify the 5'- and 3'-UTRs of small subunit ribosomal RNA (ssurRNA): 5'UTR GCCACCGCGGGAAATACGACCAATATGTAATTATTGGATAATAATTG; 5'UTR (R) - GCCCGCGGCCGCCTACTGGCAAGATCAACCAGGTTACTATATATA; 3’UTR (F) GCCACTCGAGGAGGCTTATCCTTCCTGATAAAGTG; 3’UTR

GCCCGGTACCCAAAATACTAACCCACTATGTGCAATGTGC. For whole body imaging, 7-8 weeks old C57BL/6 mice were injected with Luc-expressing $10^{5} \mathrm{WT}$ or $10^{7} \mathrm{KO}$ parasites. On day 8 post-infection, mice were injected intraperitoneally with D-luciferin substrate $(100 \mathrm{mg} / \mathrm{kg}$ animal weight in $200 \mu \mathrm{l}$ of PBS), VivoGlo (Promega, P1041), and imaged after 5 min using in vivo Imaging System IVIS Lumina XR with medium binning, 10 sec exposure and 12.5 FOV, under XGI-8 gas anesthesia system. For ex vivo imaging, transcardial perfusion was carried out for infected mice with cold PBS after injecting Dluciferin, and the organs were dissected out and imaged. 


\section{Analyses of cytokines, chemokines, chemokine receptors and other key mediators of cerebral pathogenesis}

Bio-Plex assays for cytokines and chemokines were carried out in Bio-Plex 200 system using Bio-Plex Pro Mouse Cytokine Grp I Panel 23-Plex assay kit (Bio-Rad, M60009RDPD) following manufacturer's protocol. The plasma samples utilized for the assays were prepared from the infected mouse blood collected on day $7 / 8$ post-infection. For transcript levels, total RNA was isolated from the brain samples of WT and KO-infected mice that were collected after a thorough perfusion with cold PBS. qPCR analyses were performed using QuantiFast SYBR Green RT-PCR Kit (Qiagen, 204154) on StepOne Real-Time PCR System (Applied Biosystems). The primers used were listed in Supplementary table 4. Expression levels were normalized with GAPDH and fold changes for the transcripts of KO-infected mice with respect to WT were calculated using $2^{(-\Delta \Delta C t)}$ method.

\section{Flow cytometry}

Flow cytometry analyses of T cells in the brain samples of WT- and $\mathrm{KO}$-infected mice were carried out as described ${ }^{83}$. In brief, mice were anesthetized and transcardially perfused with PBS, and the brain samples were dissected out and harvested in RPMI-1640 medium containing $10 \%$ FBS. For preparing single cell suspensions, the samples were minced and digested in RPMI-1640 medium containing $0.05 \%$ Collagenase D and $2 \mathrm{U} / \mathrm{ml}$ DNase I for 30 minutes at room temperature, and passed through $70 \mu \mathrm{m}$ nylon cell strainer, followed by 5 minutes of incubation on ice. Brain homogenates were then overlaid on $30 \%$ Percoll cushion and centrifuged at $400 \mathrm{~g}$ for 20 minutes at room temperature. The leukocyte pellets obtained were resuspended in $1 \mathrm{ml}$ of $\mathrm{RBC}$ lysis buffer $\left(155 \mathrm{mM} \mathrm{NH}_{4} \mathrm{Cl}, 10 \mathrm{mM} \mathrm{NaHCO}\right.$ and $0.1 \mathrm{mM}$ EDTA; $\mathrm{pH} 7.3$ ) and incubated on ice for 5 minutes to remove any residual RBCs. The pellets 
were then washed with RPMI-1640, counted and stained for various markers. For intracellular markers like TNF $\alpha$, IFN $\gamma$, perforin and granzyme B, staining was carried out after fixing the cells with $2 \%$ paraformaldehyde. Flow cytometry was performed with BD LSRFortessa and the data was analysed using Flow $\mathrm{Jo}_{0}^{\mathrm{TM}}$ v10.6.1. The following fluorescent dye-conjugated antibodies were used for staining: anti-mouse CD3-FITC (clone 17A2; eBioscience), anti-mouse CD4-PE (clone RM4-5; eBioscience), anti-mouse CD8-PerCPCyanine5.5 (clone 53-6.7; eBioscience), anti-mouse CD69-Brilliant Violet 421 (clone H1.2F3; BioLegend), anti-mouse CXCR3-PE (clone CXCR3-173; eBioscience), anti-mouse Perforin-PE (clone S16009A; BioLegend), anti-mouse Granzyme B-APC (clone NGZB; eBioscience), anti-mouse IFN $\gamma$-eFluor 450 (clone XMG1.2; eBioscience), anti-mouse TNF $\alpha$ eFluor 450 (clone MP6-XT22; eBioscience).

\section{FV isolation and analyses}

In $P$. berghei, the Hz containing vacuoles tend to remain as small discrete vacuoles until the late schizont stage and they coalesce only at the time of schizont rupture. Because of this, purifying the FVs for $P$. berghei trophozoites is difficult using the standard percoll protocol that is followed for $P$. falciparum, wherein the small vacuoles coalesce in the late ring stages itself. Hence, we resorted ourselves to the isolation of FVs that are released during the schizont rupture as described with slight modifications ${ }^{84}$. In brief, the infected blood containing WT or FCKO parasites was collected around 22:00 h, centrifuged at 1,000 $\mathrm{g}$ for 5 min to remove plasma and buffy coat, and washed twice with RPMI-1640 medium containing $10 \%$ FBS. The washed cells were resuspended in 10 volumes of RPMI-1640 medium containing $10 \% \mathrm{FBS}$ and then incubated at $37^{\circ} \mathrm{C}$ for overnight in a $\mathrm{CO}_{2}$ incubator. The maturation of schizonts was monitored by Giemsa smears and the FVs released in the culture supernatant during schizont rupture were collected in the next day around 09:00 h. In brief, 
the cultures were centrifuged at $200 \mathrm{~g}$ for $5 \mathrm{~min}$ to remove the RBCs. After repeating this step twice, the supernatant devoid of RBCs was centrifuged at $400 \mathrm{~g}$ for $10 \mathrm{~min}$ to collect the FVs that are free of merozoites. The FV pellet was then washed twice with PBS by centrifuging at $3000 \mathrm{~g}$ for $3 \mathrm{~min}$ and stored at $-20^{\circ} \mathrm{C}$. The purity of FVs was tested under microscope with 100x objective. Similar approach was followed for the isolation of FVs from griseofulvintreated and -untreated parasites.

\section{Microscopy analyses of LysoTracker Deep Red uptake, BODIPY 493/503 and Nile Red staining, $\mathrm{Hz}$ content and $\mathrm{Hz}$ dynamics}

To examine the LysoTracker Deep Red uptake in the live parasites, 10-20 $\mu$ l of infected blood was collected from tail vein of the infected mice when the peripheral blood parasitemia was around 5-10\% and resuspended in heparinised RPMI-1640 medium containing 10\% FBS. After washing twice, the cells were incubated with 100 nM LysoTracker Deep Red (Thermo Fisher Scientific, L12492) for $30 \mathrm{~min}$ at $37^{\circ} \mathrm{C}$ in RPMI-1640 medium containing 10\% FBS. The cells were then washed thrice with RPMI-1640 medium without phenol red, resuspended in the same medium and examined immediately under $100 \mathrm{x}$ objective using Olympus IX83 microscope with DP73 high-performance camera at 1600x1200 resolution using TRITC filter. BODIPY 493/503 (Thermo Fisher Scientific, D3922) and Nile Red (Sigma-Aldrich, 19123) staining for parasitized RBCs were carried out as described earlier ${ }^{85}$ In brief, 10-20 $\mu \mathrm{l}$ of infected mouse blood was collected from the tail vein in Hank's balanced salt solution (HBSS) and centrifuged at $2000 \mathrm{~g}$ for $3 \mathrm{~min}$. The cell pellet obtained was resuspended in $200 \mu \mathrm{l}$ of HBSS containing BODIPY 493/503 $(10 \mu \mathrm{g} / \mathrm{ml})$ or Nile Red (2 $\mu \mathrm{g} / \mathrm{ml}$ ) and incubated at $37^{\circ} \mathrm{C}$ for $30 \mathrm{~min}$. The cells were then washed twice and resuspended in HBSS. The images were acquired under 100x objective using Olympus IX83 microscope with DP73 high-performance camera at 1600x1200 resolution using FITC/TRITC filters. The 
images for WT and $\mathrm{KO}$ were acquired under identical exposure conditions and the fluorescent signal intensities were quantified using Image J software. To examine the $\mathrm{Hz}$ content, bright field and DIC images were taken under 100x objective. Live imaging of $\mathrm{Hz}$ dynamics was carried out under 100x objective by acquiring 15 frames per second at 1600x1200 resolution. The video files were processed using VSDC video editor and VideoPad by NCH softwares.

\section{Labelling studies with ${ }^{14} \mathrm{C}$-ALA, ${ }^{32} \mathrm{P}$-orthophosphoric acid and ${ }^{14} \mathrm{C}-\mathrm{SA}$}

$\left[4-{ }^{14} \mathrm{C}\right]$-ALA (ARC 1550), ${ }^{32} \mathrm{P}$-orthophosphoric acid (ARC 0103) and $\left[1-{ }^{14} \mathrm{C}\right]-\mathrm{SA}(\mathrm{ARC} 025)$ were procured from American Radiolabeled Chemicals, Inc. The infected blood samples were collected, centrifuged at $1,000 \mathrm{~g}$ for $5 \mathrm{~min}$ to remove plasma and buffy coat, and washed twice with RPMI-1640 medium containing 10\% FBS. The washed cells were resuspended in 10 volumes of RPMI-1640 medium containing 10\% FBS and then incubated at $37^{\circ} \mathrm{C}$ in a $\mathrm{CO}_{2}$ incubator with the respective radioactive compounds. For ${ }^{14} \mathrm{C}$-ALA labelling, blood samples were collected from griseofulvin treated and control WT-infected mice around 16:00 $\mathrm{h}$ and the labelling was carried out for $9 \mathrm{~h}$ at a radioactivity of $1 \mu \mathrm{Ci} / \mathrm{ml}$. The infected RBCs were then centrifuged, washed with PBS and the parasites were isolated by saponin treatment. After washing the parasite pellet with PBS for four times, free heme present in the parasites was extracted using ethylacetate:glacial acetate (4:1) followed by 1.5 $\mathrm{N} \mathrm{HCl}$ and water washes to remove porphyrins and ALA as described earlier ${ }^{44}$. The upper phase was separated, dried under nitrogen stream, dissolved in methanol and analysed by TLC on silica gel using the mobile phase 2,6-lutidine and water $(5: 3 \mathrm{v} / \mathrm{v})$ in ammonia atmosphere. The intensity of radiolabelling was scanned using Amersham Typhoon 5 Biomolecular Imager by exposing the TLC sheets to phosphorimager screen and the

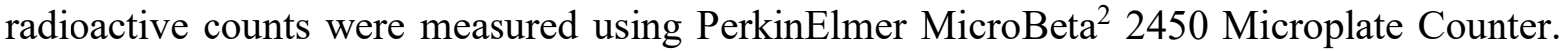


For ${ }^{32} \mathrm{P}$-orthophosphoric acid and ${ }^{14} \mathrm{C}$-SA labelling, the blood sample collection and incubation were carried out as mentioned for the FV isolation. In addition to the isolation of the secreted FVs, the infected RBC pellets were subjected to saponin treatment for the isolation of the radiolabelled total parasites. The labelling was carried out for ${ }^{32} \mathrm{P}$ orthophosphoric acid and ${ }^{14} \mathrm{C}-\mathrm{SA}$ at radioactivities of $50 \mu \mathrm{Ci} / \mathrm{ml}$ and $2 \mu \mathrm{Ci} / \mathrm{ml}$, respectively. All these experiments were typically carried out in a total volume of $4 \mathrm{ml}$, carefully matching in terms of parasitized RBCs and haematocrit between WT and FCKO.

\section{Lipid analyses}

Lipid extraction for phospholipid analysis was carried out as described ${ }^{86}$. In brief, the FV and parasite pellets were extracted with chloroform:methanol (2:1) and washed twice with water. The lower organic phase was collected and analysed by TLC on silica gel using the mobile phase chloroform:ethanol:water:tri-ethylamine (30:35:7:35 v/v). $5 \mu 1$ of the sample was used to take radioactive counts. For fatty acid analysis, the lipids extracted as mentioned above were dried, dissolved in $50 \mu \mathrm{l}$ of toluene and subjected to mild methanolysis/methylation by the addition of $375 \mu \mathrm{l}$ of methanol and $75 \mu \mathrm{l}$ of $8 \% \mathrm{HCl}$ in methanol. After incubating at $45^{\circ} \mathrm{C}$ for $16 \mathrm{~h}$, FAMEs were extracted by the addition of $250 \mu \mathrm{l}$ of hexane and $250 \mu \mathrm{l}$ of water $^{87}$. The hexane layer containing FAMEs was analysed by TLC on silica gel impregnated with $0.5 \%$ methanolic silver nitrate using the mobile phase light petroleum ether:acetone:formic acid $(97: 2: 1 \mathrm{v} / \mathrm{v})$ to separate FAMEs derived from unsaturated fatty $\operatorname{acids}^{88}$. In this separation, SAME lacking double bond migrates faster as an uppermost top band followed by OAME and other unsaturated FAMEs that are separated based on their degree of unsaturation and the length of fatty acyl chain. The respective standards were used for all the lipids. $5 \mu \mathrm{l}$ of the hexane layer was used to take radioactive counts. For GC-MS analysis, $1 \mu 1$ of hexane containing the FAMEs was injected in split mode (50:1). The GC- 
MS analysis was performed using Agilent 7890B GC coupled with 240 Ion Trap MS. The column used for analysis was Agilent VF-5MS (30 m length x $0.25 \mathrm{~mm}$ internal diameter (ID) $\mathrm{x} 0.25 \mu \mathrm{m}$ film thickness). The oven temperature was programmed from $140^{\circ} \mathrm{C}$ (5 min hold), increased at rate of $4^{\circ} \mathrm{C} / \mathrm{min}$ to $240^{\circ} \mathrm{C}$. Helium was used as carrier gas with $1 \mathrm{ml} / \mathrm{min}$ flow rate. The mass spectrometer was operated in full scan mode from 40 to $500 \mathrm{~m} / \mathrm{z}$ and NIST library search was performed to identify the compounds.

\section{Proteomics analyses}

To examine the protein content of the FVs from WT and FCKO parasites, proteins were extracted from three different preparations of WT and FCKO FVs using $25 \mathrm{mM}$ ammonium bicarbonate containing $6 \mathrm{M}$ urea, followed by treatment with DTT and iodoacetamide. The urea concentration was reduced to $0.8 \mathrm{M}$ by performing dilution with $25 \mathrm{mM}$ ammonium bicarbonate. In-solution trypsin (SCIEX) digestion was carried out for $200 \mu \mathrm{g}$ total protein, followed by desalting. LC-MS/MS analyses were performed using SCIEX TripleTOF 5600+ ESI-Mass Spectrometer. The acquired data were analysed using Paragon algorithm against Plasmodium berghei Fasta files from UniProt for 95\% confidence.

\section{Griseofulvin treatment in mice}

Griseofulvin (Sigma-Aldrich) was prepared by dissolving 1 or $2 \mathrm{mg}$ in $40 \mu \mathrm{DMSO}$ and then making up the volume to $200 \mu \mathrm{l}$ with $10 \%$ solutol HS 15 (Sigma-Aldrich) in saline. The mixture was vortexed thoroughly for $10 \mathrm{~min}$ to form an emulsion and injected intraperitoneally into the mice. All single dose injections were carried out at 06:00 $\mathrm{h}$ and double dose injections were carried out at $06: 00 \mathrm{~h}$ and 18:00 $\mathrm{h}$ for the respective days. Control mice were injected with the solvent. 


\section{Western blot analyses and other procedures}

Western analyses were carried out using standard procedures. In brief, brain samples were homogenized in $50 \mathrm{mM}$ Tris-Cl buffer, $\mathrm{pH} 7.5$ containing $5 \mathrm{mM}$ EDTA, $50 \mathrm{mM} \mathrm{NaCl}, 5 \mathrm{mM}$ DTT, 0.1\% Np-40, $50 \mathrm{mM} \mathrm{NaF}, 1 \mathrm{mM}$ PMSF, $1 \mathrm{mM} \mathrm{Na} \mathrm{VO}_{4}$, and 1x Halt Protease Inhibitor Cocktail (Thermo Fisher Scientific), followed by centrifugation at 18,000 $\mathrm{g}$ for $20 \mathrm{~min}$ at $4^{\circ} \mathrm{C}$. The supernatants were collected and quantified for total protein. The following antibodies were used: anti-mouse NF-кB p65 (Invitrogen, 14-6731-81); anti-mouse PhosphoNF-אB p65 (Ser536) (Invitrogen, MA5-15160); anti-mouse NLRP3 (Invitrogen, PA5-20838); anti-mouse Phospho-NLRP3 (Ser295) (Invitrogen, PA5-105071); anti-mouse Caspase 1 (Invitrogen, 14-9832-82); anti-mouse Cleaved Caspase-1 (Asp296) (Cell Signaling Technology, 89332); anti-mouse IL-1 (Invitrogen, \# 701304); anti-mouse Cleaved IL-1 $\beta$ (Cell Signaling Technology, 52718) and anti-mouse $\beta$-Actin (Cell Signaling Technology, 3700). Blots were developed using Pierce ECL Western Blotting Substrate (Thermo Fisher Scientific).

\section{Statistical analyses}

All the graphs were plotted using GraphPad Prism 7 software and the statistical analyses were carried out using unpaired t-test and log-rank (Mantel-Cox) test. n.s - not significant, $* P<0.05, * * P<0.01, * * * P<0.001$.

\section{FIGURE LEGENDS}

Fig. 1| CM protection in heme pathway KO parasite-infected mice. a, Growth analysis of $\operatorname{PbWT}(\mathrm{n}=10), \operatorname{PbALASKO}(\mathrm{n}=12)$ and $\operatorname{PbFCKO}(\mathrm{n}=12)$ parasites in C57BL/6 mice. $10^{5}$ parasites were used to initiate $P b \mathrm{WT}$ and $\mathrm{PbKO}$ parasite infections. The data represent three different batches. b, Mortality curves of mice infected with $P b \mathrm{WT}, P b A L A S K O$ and 
PbFCKO parasites. The data represent the mice utilized for growth curve analysis $(* * * P<0.001$, log-rank (Mantel-Cox) test). c, Spleen weight of mice infected with $P b \mathrm{WT}$ $(\mathrm{n}=13), \operatorname{PbALASKO}(\mathrm{n}=14)$ and PbFCKO $(\mathrm{n}=14)$ parasites. For each day, 3-5 mice from four different batches were included (mean $\pm \mathrm{SD} * * P<0.01, * * * P<0.001$, unpaired t-test). d, Growth analysis of $P b W T(n=14), P b A L A S K O ~(n=14)$ and PbFCKO $(\mathrm{n}=14)$ parasites in C57BL/6 mice. $10^{5}$ and $10^{7}$ parasites were used to initiate $\mathrm{WT}$ and $\mathrm{KO}$ parasite infections, respectively. The data represent four different batches. e, Mortality curves of mice infected with $P b \mathrm{WT}, P b A L A S K O$ and $P b F C K O$ parasites. The data represent the mice utilized for growth curve analysis $(* * * P<0.001, \log$-rank (Mantel-Cox) test). f, Percentage of infected reticulocytes in the parasitized red cells $\left({ }^{*} P<0.05, * * P<0.01\right.$, unpaired t-test). The data represent six mice each for $P b \mathrm{WT}, P b A L A S K O$ and $P b F C K O$ parasites. g, Giemsa-stained images for peripheral blood smears prepared from tail vein blood of $P b \mathrm{WT}$ and $\mathrm{PbKO}$ parasite-infected mice. Reticulocytes could be identified by their distinct blue color. Images were captured using 100x objective lens. Scale bar $=5 \mu \mathrm{m}$. h, RMCBS score for mice infected with $P b \mathrm{WT}(\mathrm{n}=8)$ and $P b K O(\mathrm{n}=12)$ parasites (mean $\pm \mathrm{SD} ; * * * P<0.001$, unpaired ttest).

Fig. 2| Generation of Luc-expressing heme pathway KO parasites and in vivo bioluminescence imaging of infected mice. a, Double crossover recombination strategy utilized to generate Luc-expressing PbALASKO and PbFCKO parasites. b, PCR confirmation for $A L A S$ and $F C$ deletions with genomic DNA isolated from Luc-expressing PbALASKO and PbFCKO parasites. Lane M: $1 \mathrm{~kb}$ ladder; Lane 1, 3 and 5: ALAS product (2.11 kb); Lane 2, 4 and 6: FC product (1.54 kb). c, RT-PCR confirmation for $A L A S$ and FC deletions with total RNA isolated from Luc-expressing PbALASKO and $P b F C K O$ parasites. Lane M: $1 \mathrm{~kb}$ ladder; Lane 1, 3 and 5: ALAS product (1.92 kb); Lane 2, 4 and 6: FC product 
$(1.05 \mathrm{~kb})$. d, Live GFP and m-cherry fluorescence of Luc-expressing $P b \mathrm{WT}$ and heme pathway $P b K O$ parasites. Images were captured using 100x objective lens. Scale bar $=5 \mu \mathrm{m}$. e, Whole body bioluminescence imaging of WT and KO parasite-infected mice on day 8 post-infection. f, Ex vivo bioluminescence imaging of liver (Li), lungs (Lu), brain (B), heart $(\mathrm{H})$ and spleen $(\mathrm{S})$ of Luc-expressing $\mathrm{PbWT}$ - and $\mathrm{PbKO}$-infected mice. Enlarged images of brain are shown. g, Mortality curves of mice infected with Luc-expressing $P b \mathrm{WT}(\mathrm{n}=5)$, $\operatorname{PbALASKO}(\mathrm{n}=4)$ and $P b F C K O(\mathrm{n}=5)$ parasites $(* * * P<0.001, \log$-rank (Mantel-Cox) test).

\section{Fig. 3| Assessment of cerebral pathology in heme pathway KO parasite-infected mice. a,}

Evans blue extravasation in the brain of mice infected with $P b \mathrm{WT}$ and heme pathway $\mathrm{PbKO}$ parasites. b, Quantification of Evans blue in the brain samples of mice infected with $P b \mathrm{WT}$ $(\mathrm{n}=6)$ and heme pathway $\operatorname{PbKO}(\mathrm{n}=9)$ parasites. (mean $\pm \mathrm{SD})(* * * P<0.001$, unpaired t-test). c, H\&E staining of the brain sections prepared from $P b \mathrm{WT}$ and $P b \mathrm{KO}$ parasite-infected mice. Black arrows - intracerebral and petechial hemorrhages, blue arrows - thrombosed blood vessels and brown arrows - gross demyelination. Images were captured using 10x objective lens. Scale bar $=50 \mu \mathrm{m}$. d, IgG extravasation in the brain sections of $\mathrm{PbWT}$ and $\mathrm{PbKO}$ parasite-infected mice. Black arrows - areas showing IgG immunoreactivity. Images were captured using 10x objective lens. Scale bar $=50 \mu \mathrm{m}$. e, H\&E staining of the brain sections indicating (black arrows) occluded vasculatures containing luminal and abluminal leukocytes, and parasite-derived Hz. Images were captured using 60x objective lens. Scale bar $=10 \mu \mathrm{m}$. f, Immunofluorescence analysis of parasite accumulation in the brain sections of $P b \mathrm{WT}$ and $\mathrm{PbKO}$ parasite-infected mice. g, Immunofluorescence analysis of $\mathrm{CD}^{+}$cells in the blood vessels of $P b \mathrm{WT}$ and $P b K O$ parasite-infected mice. $\mathbf{h}$, Immunofluorescence analysis of $\beta$ APP staining in the brain sections of $P b \mathrm{WT}$ and $\mathrm{PbKO}$ parasite-infected mice. Images were captured using 20x objective lens. Scale bar $=20 \mu \mathrm{m}$. 
Fig. 4| Assessment of inflammatory parameters in heme pathway KO parasite-infected mice. a, Plasma cytokine and chemokine levels of $P b \mathrm{WT}$ - and $P b K O$-infected mice $(\mathrm{n}=5)$ (mean $\pm \mathrm{SD} ; * P<0.05, * * P<0.01, * * * P<0.001$, unpaired t-test). b, qPCR analyses of host transcripts in the brain samples of infected mice. Expression levels were normalized with mouse GAPDH. Relative expression fold changes of mRNA transcripts in the KO-infected mice with respect to WT-infected mice (mean $\pm \mathrm{SD})$ are shown $(\mathrm{n}=3)$. c, Flow cytometry analyses of $\mathrm{T}$ cells in the brain samples of infected mice. Mice on day $7 / 8$ post-infection were used and the data for each cell type were obtained from three different mice infected with $P b \mathrm{WT}$ or $P b \mathrm{KO}$ parasites (mean $\pm \mathrm{SD} ; * * P<0.01, * * * P<0.001$, unpaired t-test). d, Western analyses of brain homogenates prepared from $P b \mathrm{WT}$ - and PbKO-infected mice. $200 \mu \mathrm{g}$ of total protein was used from the pooled brain homogenates of three different mice for $P b \mathrm{WT}$, PbALASKO and $P b$ FCKO.

Fig. 5| $\mathrm{Hz}$ and heme levels in KO parasites. a,b, Bright field images of Giemsa-stained $\mathrm{PbWT}, \mathrm{PbALASKO}$ and PbFCKO asexual stage parasites and gametocytes, respectively, showing Hz content. Images were captured using 100x objective lens. Scale bar $=5 \mu \mathrm{m}$. c, $\mathrm{Hz}$ content in differential interference contrast (DIC; left) and bright field images (right) of paraformaldehyde-fixed $\mathrm{pRBCs}$ containing $\mathrm{PbWT}$ and $\mathrm{PbKO}$ parasites. Images were captured using 100x objective lens. Scale bar $=5 \mu \mathrm{m} . \mathbf{d}, \mathrm{Hz}$ levels in $\mathrm{PbWT}$ and $\mathrm{PbKO}$ parasites e, Free heme levels in $P b \mathrm{WT}$ and $P b \mathrm{KO}$ parasites. f, Free heme levels in the plasma samples of $P b \mathrm{WT}$ and $P b \mathrm{KO}$ parasite-infected mice. g, Heme/Hemopexin ratio in the plasma samples of $P b \mathrm{WT}$ and $P b \mathrm{KO}$ parasite-infected mice. h, Plasma hemopexin levels of $P b \mathrm{WT}$ and $\mathrm{PbKO}$ parasite-infected mice. i, Plasma hemoglobin levels of $P b \mathrm{WT}$ and $P b \mathrm{KO}$ parasiteinfected mice. $\mathbf{j}, \mathbf{k}, \mathrm{Hz}$ load in the spleen and liver of $\mathrm{PbWT}$ and $\mathrm{PbKO}$ parasite infected mice, 
respectively. c-k, The data represent 9 mice each for $P b \mathrm{WT}, \mathrm{PbALASKO}$ and $\mathrm{PbFCKO}$. (mean $\pm \mathrm{SD} ;$ n.s - not significant, $* P<0.05,{ }^{* *} P<0.01,{ }^{* * *} P<0.001$, unpaired t-test).

Fig. 6| Sensitivity of WT and FCKO parasites to $\alpha, \beta$-arteether and chloroquine. a,b, Blood parasitemia and mortality curves of infected mice treated with $\alpha, \beta$-arteether, respectively. The data represent four mice for each group (n.s - not significant, log-rank (Mantel-Cox) test). c,d, Blood parasitemia and mortality curves of infected mice treated with chloroquine, respectively. The data represent four mice for each group $(* * P<0.01, \log$-rank (Mantel-Cox) test).

Fig. 7| Assessment of food vacuole pH, phospholipids and neutral lipids in FCKO parasites. a, Live cell fluorescence imaging of LysoTracker Deep Red uptake in $P b \mathrm{WT}$ and PbFCKO parasites. b, Quantification of fluorescence signal from various stages. $P b \mathrm{WT}-98$ trophozoites and 22 schizonts; PbFCKO - 95 trophozoites and 23 schizonts. ET, MT and LT early, mid and late trophozoites; S - schizonts. Images were captured using 100x objective lens. Scale bar $=5 \mu \mathrm{m}$. (mean $\pm \mathrm{SD} ;{ }^{* * *} P<0.001$, unpaired t-test). c, TLC separation of ${ }^{32} \mathrm{P}-$ orthophosphoric acid radiolabelled phospholipids for $\mathrm{PbWT}$ and $\mathrm{PbFCKO}$ total parasites and FVs. Half the phospholipid preparation was used for total parasites. For FVs, entire preparation was used. PC - phosphatidylcholine; PI - phosphatidylinositol; PE phosphatidylethanolamine. d, Band intensities quantified (mean $\pm \mathrm{SD} ; \mathrm{n} . \mathrm{s}$ - not significant, unpaired t-test). The data represent three different experiments. e, Live cell fluorescence imaging of BODIPY 493/503 staining in WT and FCKO parasites. Images were captured using 100x objective lens. Scale bar $=5 \mu \mathrm{m}$. f, Live cell fluorescence imaging of Nile Red staining in WT and FCKO parasites. Images were captured using 100x objective lens. Scale bar $=5 \mu \mathrm{m} . \mathbf{g}$, Quantification of the fluorescence signal from various stages for BODIPY 
493/503 staining. The data represent 69 trophozoites and 15 schizonts for $P b \mathrm{WT}$, and 68 trophozoites and 14 schizonts for $\mathrm{PbFCKO}$ (mean $\pm \mathrm{SD}$; n.s - not significant, unpaired t-test). h, Quantification of the fluorescence signal from various stages for Nile Red staining. The data represent 68 trophozoites and 14 schizonts for $P b \mathrm{WT}$, and 70 trophozoites and 14 schizonts for PbFCKO (mean $\pm \mathrm{SD}$; n.s - not significant, unpaired t-test). ET - early trophozoites; MT - mid trophozoites; LT - late trophozoites; S - schizonts.

Fig. 8| Evaluation of OA synthesis and FV proteomics for WT and FCKO parasites. a, TLC separation of ${ }^{14} \mathrm{C}-\mathrm{SA}$ radiolabelled unsaturated FAMEs for $\mathrm{PbWT}$ and $\mathrm{PbFCKO}$ total parasites and FVs. One third of the FAME preparation used for total parasites and entire preparation used for FVs. b,c, Band intensities quantified for total parasites and FVs, respectively (mean $\pm \mathrm{SD}$; n.s - not significant, $* P<0.05, * * P<0.01, * * * P<0.001$, unpaired ttest), from three different experiments. d, Venn diagram of total proteins identified in the $\mathrm{PbWT}$ and $\mathrm{PbFCKO}$ FVs. e, Functional classification of the proteins based on gene ontologies available at PlasmoDB and UniProt databases. Supplementary Table 2 and Supplementary Table 3 have the complete set of details related to $\mathrm{PbWT}$ and $\mathrm{PbFCKO} F \mathrm{~F}$ proteome analyses.

Fig. 9| Effect of griseofulvin treatment on CM pathogenesis. a, Mortality curves of $P b W T$ infected mice treated with different dosages of griseofulvin $(* * * P<0.001$, log-rank (MantelCox) test). b, Growth curve analysis $(\mathrm{n}=12)$ for $2 \mathrm{mg}$ dose per day on day 4,5,6,7 and 8. c, Phosphorimager and scanned images of TLC performed for ${ }^{14} \mathrm{C}$-ALA labelled parasite free heme and the radioactive counts measured for three different experiments (mean $\pm \mathrm{SD}$; n.s not significant, $* P<0.05$, unpaired t-test). d, Extravasation of Evans blue and its quantification $(\mathrm{n}=3)($ mean $\pm \mathrm{SD} ; * * * P<0.001$, unpaired t-test). e, H\&E staining of the brain 
sections. Images were captured using $10 \mathrm{x}$ objective lens. Scale bar $=50 \mu \mathrm{m}$. f, Parasite accumulation $\mathbf{g}, \mathrm{CD}^{+}$cells in the blood vessels. $\mathbf{h}$, Axonal injury in the brain sections. Images were captured using $20 \mathrm{x}$ objective lens. Scale bar $=20 \mu \mathrm{m}$. i, Giemsa-stained parasites. Images were captured using 100x objective lens. Scale bar $=5 \mu \mathrm{m}$. j, Hz content in differential interference contrast (DIC; left) and bright field images (right) of paraformaldehyde-fixed RBCs. Images were captured using 100x objective lens. Scale bar $=$ $5 \mu m . \mathbf{k}$, Parasite $\mathrm{Hz}(\mathrm{n}=5)$. $\mathbf{l}$, Parasite free heme $(n=5) . \mathbf{m}$, Plasma free heme $(n=6) . \mathbf{n}$, Plasma hemopexin $(n=6)$. o, Plasma heme/hemopexin ratio. p, Plasma hemoglobin $(n=11)$. kp, $\left(\right.$ mean $\pm \mathrm{SD} ; \mathrm{n} . \mathrm{s}-$ not significant, ${ }^{* *} P<0.01, * * * P<0.001$, unpaired t-test $)$.

Fig. 10| Model depicting the role of de novo heme in regulating $\mathrm{FV}$ integrity and $\mathrm{Hz}$ formation. De novo heme pathway of malaria parasite and $\mathrm{Hz}$ formation in the $\mathrm{FV}$ are represented. Solid arrows indicate the effect of de novo heme on V-type $\mathrm{H}^{+}$-ATPase, FV pH and OA synthesis affecting hemozoin formation. Dashed arrows represent the probable effects of de novo heme on $\mathrm{Hb}$ endocytosis, and protein and lipid trafficking. Gly - glycine; Succ-coA - succinyl coenzymeA; ALA - $\delta$-aminolevulinic acid; PBG - prophobilinogen; URogenIII - uroporphyrinogen III; CPogenIII - coproporphyrinogen III; PPogenIII protoporphyrinogen III; PPIX - protoporphyrin IX; ER - endoplasmic reticulum.

\section{SUPPLEMENTARY INFORMATION}

\section{Supplementary Figure 1: Growth characterization of heme pathway KO parasites in}

Balb/c mice. a, Growth analysis in Balb/c mice $(n=6) .10^{5}$ parasites were used to initiate parasite infections. $\mathbf{b}$, Mortality curves $(\mathrm{n}=6)(* * * P<0.001$, log-rank (Mantel-Cox) test). 
Supplementary Figure 2: Dot plots from flow cytometry analyses representing the $T$ cells in the brain samples of $\mathbf{W T}$ and KO-infected mice. The data represent $\mathrm{CD}^{+} \mathrm{CD} 4^{+}$ and $\mathrm{CD}^{+} \mathrm{CD}^{+}$double positive cells, and $\mathrm{CD} 3^{+} \mathrm{CD}^{+} \mathrm{CD}^{+} 9^{+}, \mathrm{CD}^{+} \mathrm{CD}^{+} \mathrm{CXCR}^{+}, \mathrm{CD}^{+}$ $\mathrm{CD}^{+}$perforin $^{+}, \mathrm{CD}^{+} \mathrm{CD}^{+}$granzyme $\mathrm{B}^{+}, \mathrm{CD}^{+} \mathrm{CD}^{+} \mathrm{TNF}^{+}$and $\mathrm{CD}^{+} \mathrm{CD}^{+} \mathrm{IFN}^{+}$triple positive cells, obtained for WT-, ALASKO- and FCKO-infected mice.

Supplementary Figure 3: Hz and free heme levels in WT and FCKO parasites isolated from Balb/c mice. a,b, Hz and free heme levels in WT and FCKO parasites $(n=3)$, respectively (mean $\pm \mathrm{SD} * P<0.05, * * P<0.01$, unpaired t-test).

Supplementary Figure 4: Assessment of parasite load in $\alpha, \beta$-arteether and chloroquine treated WT- and KO-infected mice. a, qPCR analyses of parasite load using PbGAPDH primers for the infected mice treated with $1 \mathrm{mg} /$ mouse dose of $\alpha, \beta$-arteether with total RNA isolated from the whole blood. The amplification curves represent primer dimers indicating the absence of detectable parasites. b, qPCR analyses for mouse GAPDH control. c, qPCR analyses of parasite load using $P b G A P D H$ primers for the infected mice treated with 0.4 $\mathrm{mg} /$ mouse dose of $\alpha, \beta$-arteether. d, qPCR analyses for mouse GAPDH control. The $\Delta C_{t}$ value obtained for FCKO with respect to WT for $0.4 \mathrm{mg}$ dosage was $-1.43 \pm 0.16$ (mean \pm SD). e, qPCR analyses of parasite load using PbGAPDH primers for the infected mice treated with $25 \mathrm{mg} / \mathrm{kg}$ dose of chloroquine. f, qPCR analyses for mouse GAPDH control. g, qPCR analyses of parasite load using $P b G A P D H$ primers for the infected mice treated with 10 $\mathrm{mg} / \mathrm{kg}$ dose of chloroquine. $\mathbf{h}, \mathrm{qPCR}$ analyses for mouse GAPDH control. The $\Delta C_{t}$ values obtained for FCKO with respect to WT were $4.02 \pm 0.91$ and $4.19 \pm 0.43$ for $25 \mathrm{mg} / \mathrm{kg}$ and $10 \mathrm{mg} / \mathrm{kg}$ doses, respectively (mean $\pm \mathrm{SD}$ ). The $C_{t}$ values obtained for mouse GAPDH were 
comparable between WT- and FCKO-infected mice. 1,2 - WT-infected mice; 3,4 - FCKOinfected mice.

Supplementary Figure 5: Radiolabelling of phospholipids in WT and FCKO parasites. Radioactive counts for ${ }^{32} \mathrm{P}$-orthophosphoric acid radiolabelled phospholipids of WT and FCKO total parasites and FVs. The data represent three different experiments (mean $\pm \mathrm{SD}$; n.s - not significant, unpaired t-test).

Supplementary Figure 6: FAMEs prepared from $\boldsymbol{P b}$ FVs. a, GC-MS analysis of FAMEs prepared from P. berghei FVs. The chromatogram peaks for the methyl esters of oleic acid and arachidonic acid and their mass spectra are shown. The entire set of compounds that could be identified and their respective area of peaks are given in Table S2. b, Radioactive counts for ${ }^{14} \mathrm{C}$-SA radiolabelled FAMEs of WT and FCKO total parasites and FVs. (mean \pm $\mathrm{SD}$; n.s - not significant, ${ }^{*} \mathrm{P}<0.05$, unpaired t-test $)$

\section{Supplementary Table 1: List of compounds identified in GC-MS analysis of the FAMEs} prepared from $\boldsymbol{P b}$ FVs. Methyl esters of oleic acid and arachidonic acid are highlighted in green. The compounds are listed in the order of their retention time.

Supplementary Table 2: $P b W T$ FV proteome and gene ontology clustering. Proteins that are identified only in WT FVs, but not in FCKO FVs are highlighted in green. The entire peptide summary along with the identified sequences is also provided. 
Supplementary Table 3: PbFCKO FV proteome and gene ontology clustering. Proteins that are identified only in FCKO FVs, but not in WT FVs are highlighted in blue. The entire peptide summary along with the identified sequences is also provided.

Supplementary Table 4: Details of the primers used for qPCR analyses of the host transcripts in the brain samples of WT- and KO-infected mice.

Supplementary Movie 1: Live imaging of hemozoin dynamics in PbWT parasite.

Supplementary Movie 2: Live imaging of hemozoin dynamics in PbALASKO parasite.

Supplementary Movie 3: Live imaging of hemozoin dynamics in PbFCKO parasite.

\section{REFERENCES}

1) World Health Organization. World Malaria Report (2020). https://www.mmv.org/sites/default/files/uploads/docs/publications/World_Malaria_Report_2

\section{$\underline{020 . p d f}$}

2) World Health Organization, Severe malaria. Trop. Med. Int. Health. 19, 7-131 (2014).

3) Zimmerman G. A. \& Castro-Faria-Neto, H. Persistent cognitive impairment after cerebral malaria: models, mechanisms and adjunctive therapies. Expert Rev. Anti. Infect. Ther. 8, 1209-1212 (2014).

4) Varo, R. et al. Adjunctive therapy for severe malaria: a review and critical appraisal. Malar. J. 17, 47 (2018). 
5) de Souza, J. B., Hafalla, J. C., Riley, E. M. \& Couper, K. N. Cerebral malaria: why experimental murine models are required to understand the pathogenesis of disease. Parasitology 137, 755-772 (2010).

6) Ghazanfari, N., Mueller, S. N. \& Heath, W. R. Cerebral malaria in mouse and man. Front. Immunol. 9, 2016 (2018).

7) Brown, H. et al. Evidence of blood-brain barrier dysfunction in human cerebral malaria. Neuropathol. Appl. Neurobiol. 25, 331-340 (1999).

8) Silamut, K. et al. A quantitative analysis of the microvascular sequestration of malaria parasites in the human brain. Am. J. Pathol. 155, 395-410 (1999).

9) Pongponratn, E. et al. An ultrastructural study of the brain in fatal Plasmodium falciparum malaria. Am J Trop Med Hyg. 69, 345-359 (2003).

10) Franke-Fayard, B., Fonager, J., Braks, A., Khan, S. M. \& Janse, C. J. Sequestration and tissue accumulation of human malaria parasites: can we learn anything from rodent models of malaria? PLoS Pathog. 6, e1001032 (2010).

11) Baptista, F. G. et al. Accumulation of Plasmodium berghei-infected red blood cells in the brain is crucial for the development of cerebral malaria in mice. Infect. Immun. 78, 40334039 (2010).

12) Nacer, A. et al. Neuroimmunological blood brain barrier opening in experimental cerebral malaria. PLoS Pathog. 8, e1002982 (2012).

13) Strangward, P. et al. A quantitative brain map of experimental cerebral malaria pathology. PLoS Pathog. 13, e1006267 (2017).

14) Dunst, J., Kamena, F. \& Matuschewski, K. Cytokines and chemokines in cerebral malaria pathogenesis. Front. Cell Infect. Microbiol. 7, 324 (2017). 
15) Grau, G. E. R. \& Wassmer, S. C. Pathogenetic immune responses in cerebral malaria. In: Malaria: Immune Response to Infection and Vaccination. (Springer International Publishing, Cham, 2017), pp. 67-80.

16) Newton, C. R. et al. Intracranial pressure in African children with cerebral malaria. Lancet 337, 573-576 (1991).

17) Newton, C. R. et al. Brain swelling and ischaemia in Kenyans with cerebral malaria. Arch. Dis. Child. 70, 281-287 (1994).

18) Medana, I. M. et al. Axonal injury in cerebral malaria. Am. J. Pathol. 160, 655-666 (2002).

19) Penet, M. F. et al. Imaging experimental cerebral malaria in vivo: significant role of ischemic brain edema. J. Neurosci. 25, $7352-7358$ (2005).

20) Taylor, T. E. et al. Differentiating the pathologies of cerebral malaria by postmortem parasite counts. Nat. Med. 10, 143-145 (2004).

21) Idro, R., Marsh, K., John, C. C. \& Newton, C. R. J. Cerebral malaria: mechanisms of brain injury and strategies for improved neurocognitive outcome. Pediatr. Res. 68, 267-274 (2010).

22) Dorovini-Zis, K. et al. The neuropathology of fatal cerebral malaria in Malawian children. Am. J. Pathol. 178, 2146-2158 (2011).

23) Milner Jr, D. A. et al. Supraorbital postmortem brain sampling for definitive quantitative confirmation of cerebral sequestration of Plasmodium falciparum parasites. J. Infect. Dis. 205, 1601-1606 (2012).

24) Jaramillo M. et al. Hemozoin-inducible proinflammatory events in vivo: potential role in malaria infection. J Immunol. 172, 3101-3110 (2004).

25) Olivier, M., Van Den Ham, K., Shio, M. T., Kassa, F. A. \& Fougeray, S. Malarial pigment hemozoin and the innate inflammatory response. Front. Immunol. 5, 25 (2014). 
26) Gowda, D. C. \& Wu, X. Parasite recognition and signaling Mechanisms in innate immune responses to malaria. Front. Immunol. 19, 9:3006 (2018).

27) Raulf, M. K. et al. The C-type lectin receptor CLEC12A recognizes Plasmodial hemozoin and contributes to cerebral malaria development. Cell Rep. 28, 30-38.e5 (2019).

28) Eugenin, E. A., Martiney, J.A. \& Berman, J. W. The malaria toxin hemozoin induces apoptosis in human neurons and astrocytes: Potential role in the pathogenesis of cerebral malaria. Brain Res. 1720, 146317 (2019).

29) Pamplona, A. et al. Heme oxygenase-1 and carbon monoxide suppress the pathogenesis of experimental CM. Nat. Med. 13, 703-710 (2007).

30) Ferreira, A., Balla, J., Jeney, V., Balla, G. \& Soares, M. P. A central role for free heme in the pathogenesis of severe malaria: the missing link? J. Mol. Med. (Berl). 86, 1097-1111 (2008).

31) Ferreira, A. et al. Sickle hemoglobin confers tolerance to Plasmodium infection. Cell 145, 398-409 (2011).

32) Jani, D. et al. HDP-a novel heme detoxification protein from the malaria parasite. PLoS Pathog. 4, e1000053 (2008).

33) Dorn, A., Stoffel, R., Matile, H., Bubendorf, A. \& Ridley, R. G. Malarial haemozoin/beta-haematin supports haem polymerization in the absence of protein. Nature 374, 269-271 (1995).

34) Sullivan Jr, D. J., Gluzman, I. Y. \& Goldberg, D. E. Plasmodium hemozoin formation mediated by histidine-rich proteins. Science 27, 219-222 (1996).

35) Bendrat, K., Berger, B. J. \& Cerami, A. Haem polymerization in malaria. Nature 378, 138-139 (1995). 
36) Fitch, C. D., Cai, G. Z., Chen, Y. F. \& Shoemaker, J. D. Involvement of lipids in ferriprotoporphyrin IX polymerization in malaria. Biochim. Biophys. Acta. 1454, 31-37 (1999).

37) Amodu, O. K., Adeyemo, A. A., Olumese, P. E. \& Gbadegesin, R. A. Intraleucocytic malaria pigment and clinical severity of malaria in children. Trans. R. Soc. Trop. Med. Hyg. 92, 54-56 (1998).

38) Newton, C. R., Taylor, T. E. \& Whitten R. O. Pathophysiology of fatal falciparum malaria in African children. Am. J. Trop. Med. Hyg. 58, 673-683 (1998).

39) Birhanu, M. et al. Hematological parameters and hemozoin-containing leukocytes and their association with disease severity among malaria infected Cchildren: A cross-sectional study at Pawe general hospital, Northwest Ethiopia. Interdiscip. Perspect. Infect. Dis. 2017, 8965729 (2017).

40) Dalko, E. et al. Multifaceted role of heme during severe Plasmodium falciparum infections in India. Infect. Immun. 83, 3793-3799 (2015).

41) Elphinstone R. E. et al. Dysregulation of the haem-haemopexin axis is associated with severe malaria in a case-control study of Ugandan children. Malaria J. 14, 511 (2015).

42) Dondorp, A. M. et al. Artesunate versus quinine in the treatment of severe falciparum malaria in African children (AQUAMAT): an open-label, randomised trial. Lancet 376, 1647-5167 (2010).

43) Surolia, N. \& Padmanaban, G. De novo biosynthesis of heme offers a new chemotherapeutic target in the human malarial parasite. Biochem. Biophys. Res. Commun. 187, 744-750 (1992).

44) Nagaraj, V. A. et al. Malaria parasite-synthesized heme is essential in the mosquito and liver stages and complements host heme in the blood stages of infection. PLoS Pathog. 9, e1003522 (2013) 
45) Nagaraj, V. A. \& Padmanaban, G. Insights on Heme Synthesis in the Malaria Parasite. Trends Parasitol. 33, 583-586 (2017).

46) Ke, H. et al. The heme biosynthesis pathway is essential for Plasmodium falciparum development in mosquito stage but not in blood stages. J. Biol. Chem. 289, 34827-34837 (2014).

47) Sigala, P. A., Crowley, J. R., Henderson, J. P. \& Goldberg, D. E. Deconvoluting heme biosynthesis to target blood-stage malaria parasites. Elife 14, 4 (2015).

48) Bonday, Z. Q., Dhanasekaran, S., Rangarajan, P.N. \& Padmanaban, G. Import of host delta-aminolevulinate dehydratase into the malarial parasite: identification of a new drug target. Nat. Med. 6, 898-903 (2000).

49) Dhanasekaran, S., Chandra, N. R., Sagar, B. K. C., Rangarajan, P. N. \& Padmanaban, G. Delta-aminolevulinic acid dehydratase from Plasmodium falciparum: indigenous versus imported. J. Biol. Chem. 279, 6934-6942 (2004).

50) Varadharajan, S., Dhanasekaran, S., Bonday, Z. Q., Rangarajan, P. N. \& Padmanaban, G. Involvement of delta-aminolaevulinate synthase encoded by the parasite gene in de novo haem synthesis by Plasmodium falciparum. Biochem. J. 367, 321-327 (2002).

51) Nagaraj, V. A. et al. Unique properties of Plasmodium falciparum porphobilinogen deaminase. J. Biol. Chem. 283, 437-444 (2008).

52) Nagaraj, V. A. et al. Localisation of Plasmodium falciparum uroporphyrinogen III decarboxylase of the heme-biosynthetic pathway in the apicoplast and characterisation of its catalytic properties. Int. J. Parasitol. 39, 559-568 (2009).

53) Nagaraj, V. A., Prasad, D., Rangarajan, P. N. \& Padmanaban, G. Mitochondrial localization of functional ferrochelatase from Plasmodium falciparum. Mol. Biochem. Parasitol. 168, 109-112 (2009). 
54) Nagaraj, V. A., Arumugam, R., Prasad, D., Rangarajan, P. N. \& Padmanaban, G. Protoporphyrinogen IX oxidase from Plasmodium falciparum is anaerobic and is localized to the mitochondrion. Mol. Biochem. Parasitol. 174, 44-52 (2010).

55) Nagaraj, V. A., Prasad, D., Arumugam, R., Rangarajan, P.N. \& Padmanaban, G. Characterization of coproporphyrinogen III oxidase in Plasmodium falciparum cytosol. Parasitol. Int. 59, 121-127 (2010).

56) Padmanaban, G., Nagaraj, V. A. \& Rangarajan, P. N. Unique Features of heme biosynthesis in the malaria parasite. In: Handbook of Porphyrin Science. With Applications to Chemistry, Physics, Materials Science, Engineering, Biology and Medicine. (World Scientific Publishing, New Jersey, 2013), vol. 27, pp. 168-205.

57) Harding, C. R. et al. Genetic screens reveal a central role for heme biosynthesis in artemisinin susceptibility. Nat. Commun. 11, 4813 (2020).

58) Olafson, K.N., Ketchum, M.A., Rimer, J.D. \& Vekilov, P.G. Mechanisms of hematin crystallization and inhibition by the antimalarial drug chloroquine. Proc. Natl. Acad. Sci. U.S.A. 112, 4946-4951 (2015).

59) Schwarzer, E., Kuhn, H., Valente, E. \& Arese, P. Malaria-parasitized erythrocytes and hemozoin nonenzymatically generate large amounts of hydroxy fatty acids that inhibit monocyte functions. Blood 101, 722-728 (2003).

60) Stiebler, R. et al. Unsaturated glycerophospholipids mediate heme crystallization: Biological Implications for hemozoin formation in the kissing bug Rhodnius prolixus. PLoS One 9, e88976 (2014).

61) Gratraud, P. et al. Oleic acid biosynthesis in Plasmodium falciparum: characterization of the stearoyl-CoA desaturase and investigation as a potential therapeutic target. PLoS One 4, e6889 (2009). 
62) Kloehn, J., Harding, C. R. \& Soldati-Favre, D. Supply and demand-heme synthesis, salvage and utilization by Apicomplexa. FEBS J. 12 June 2020 (10.1111/febs.15445).

63) Lamarque, M. et al. FV proteome of the malarial parasite Plasmodium falciparum. Proteomics Clin Appl. 2, 1361-1374 (2008).

64) Hayashi, M. et al. Vacuolar $\mathrm{H}^{(+)}$-ATPase localized in plasma membranes of malaria parasite cells, Plasmodium falciparum, is involved in regional acidification of parasitized erythrocytes. J. Biol. Chem. 275, 34353-34358 (2000).

65) Lin, J. et al. Replication of Plasmodium in reticulocytes can occur without hemozoin formation, resulting in chloroquine resistance. J. Exp. Med. 212, 893-903 (2015).

66) Hopp, C. S. et al. Deletion of the rodent malaria ortholog for falcipain-1 highlights differences between hepatic and blood stage merozoites. PLoS Pathog. 13, e1006586 (2017). 67) World Health Organization. WHO Model Prescribing Information: Drugs Used in Skin Diseases. https://apps.who.int/iris/bitstream/handle/10665/41975/9241401060.pdf?sequence=1\&isAllo $\underline{\text { wed }=\mathrm{y}}$

68) Petersen, A. B., Ronnest, M. H., Larsen, T. O. \& Clausen M. H. The chemistry of griseofulvin. Chem. Rev. 114, 12088-12107 (2014).

69) Sahoo, A. K. \& Mahajan R. Management of tinea corporis, tinea cruris, and tinea pedis: A comprehensive review. Indian Dermatol Online J. 7, 77-86 (2016).

70) Liu, K. et al. A metabolomic perspective of griseofulvin-induced liver injury in mice. Biochem. Pharmacol. 98, 493-501 (2015).

71) Jaramillo, M., Godbout, M. \& Olivier, M. Hemozoin induces macrophage chemokine expression through oxidative stress-dependent and -independent mechanisms. J. Immunol. 174, 475-484 (2005). 
72) Mandala W. L. et al. Cytokine profiles in Malawian children presenting with uncomplicated Malaria, severe malarial anemia, and cerebral malaria. Clin. Vaccine Immunol. 24, e00533-16 (2017).

73) Sorensen E. W. et al. CXCL10 stabilizes T cell-brain endothelial cell adhesion leading to the induction of cerebral malaria. JCI Insight 3, 98911 (2018).

74) Deroost, K., Pham, T.T., Opdenakker, G. \& Van den Steen, P. E. The immunological balance between host and parasite in malaria. FEMS Microbiol Rev 40, 208-257 (2016).

75) Abshire, J.R., Rowlands, C.J., Ganesan, S.M., So, P.T. \& Niles, J.C. Quantification of labile heme in live malaria parasites using a genetically encoded biosensor. Proc. Natl. Acad. Sci. U S A. 114, E2068-E2076 (2017).

76) Oh, Y. J. et al. Cytochrome b5 reductase 1 triggers serial reactions that lead to iron uptake in plants. Mol. Plant 9, 501-513 (2016).

77) Antonny, B., Vanni, S., Shindou H. \& Ferreira, T. From zero to six double bonds: phospholipid unsaturation and organelle function. Trends Cell Biol. 25, 427-436 (2015).

78) Smith, C. M. et al. Griseofulvin impairs intraerythrocytic growth of Plasmodium falciparum through ferrochelatase inhibition but lacks activity in an experimental human infection study. Sci. Rep. 7, 41975 (2017).

79) Carroll, R. W. et al. A rapid murine coma and behavior scale for quantitative assessment of murine cerebral malaria. PLoS One 5, pii: e13124 (2010).

80) Tripathi, A. K., Khan, S. I., Walker, L. A. \& Tekwani, B. L. Spectrophotometric determination of de novo hemozoin/beta-hematin formation in an in vitro assay. Anal. Biochem. 325, 85-91 (2004).

81) Deroost, K. et al. Improved methods for haemozoin quantification in tissues yield organand parasite-specific information in malaria-infected mice. Malar. J. 11, 166 (2012). 
82) Dende, C. et al. Nanocurcumin is superior to native curcumin in preventing degenerative changes in Experimental Cerebral Malaria. Sci. Rep. 7, 10062 (2017).

83) Ryg-Cornejo, V., Ioannidis, L. J. \& Hansen, D. S. Isolation and analysis of brainsequestered leukocytes from Plasmodium berghei ANKA-infected mice. J. Vis. Exp. 71, $50112(2013)$.

84) Dasari, P. et al. Digestive vacuoles of Plasmodium falciparum are selectively phagocytosed by and impair killing function of polymorphonuclear leukocytes. Blood 118, 4946-4956 (2011).

85) Palacpac, N. M. Q. et al. Developmental-stage-specific triacylglycerol biosynthesis, degradation and trafficking as lipid bodies in Plasmodium falciparum-infected erythrocytes. J. Cell. Sci. 117, 1469-1480 (2004).

86) Deranieh, R. M., Joshi, A. S. \& Greenberg, M. L. Thin-Layer Chromatography of Phospholipids. Methods Mol. Biol. 1033, 21-27 (2013).

87) Ichihara, K. \& Fukubayashi, Y. Preparation of fatty acid methyl esters for gas-liquid chromatography. J. Lipid Res. 51, 635-640 (2010).

88) Nikolova-Damyanova, B. \& Momchilova, S. Silver ion thin-layer chromatography of fatty acids. A survey. J. Liq. Chromatogr. Relat. Technol. 24, 1447-1466. (2001). 


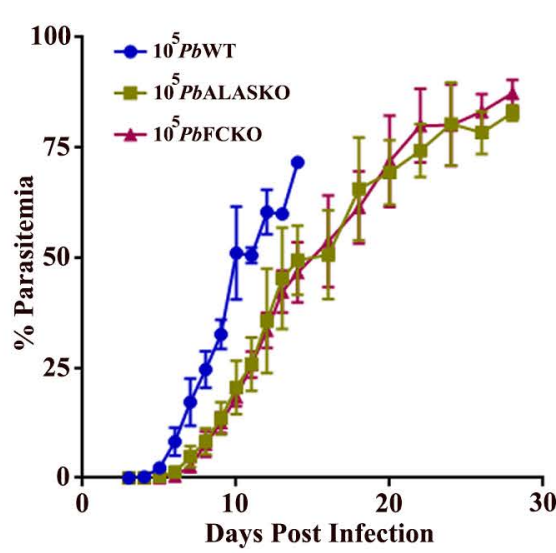

d

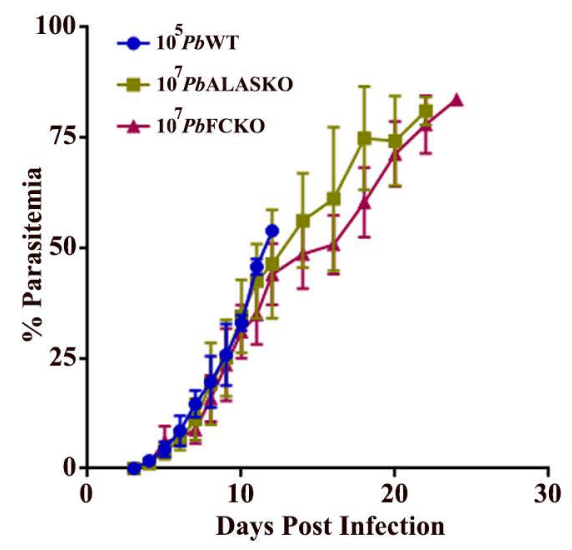

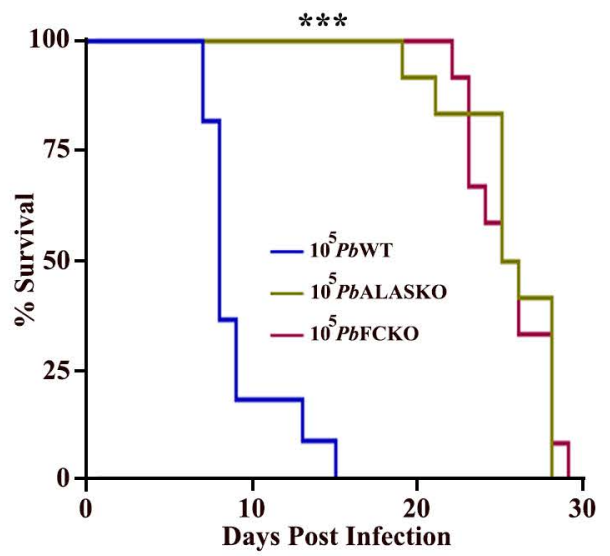

e

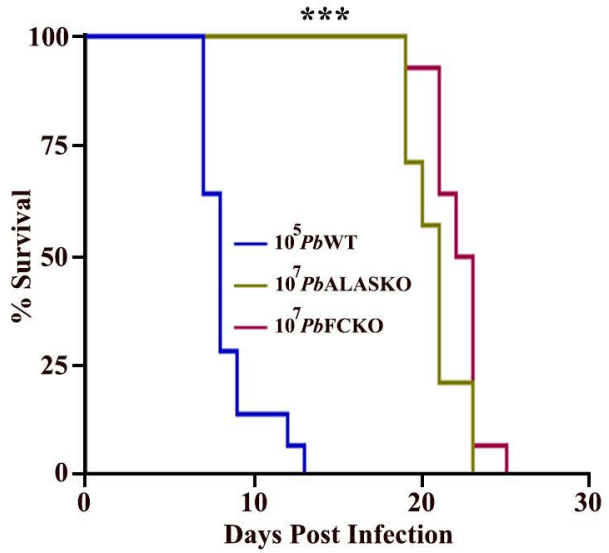

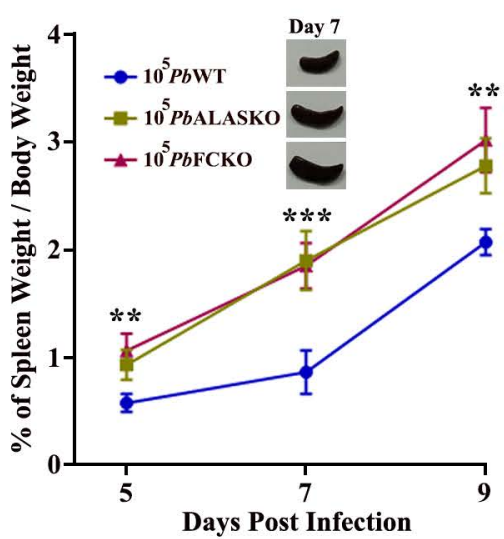

f

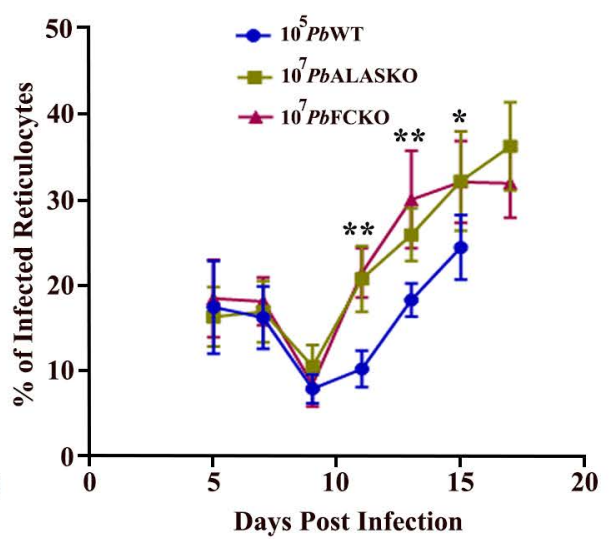

g

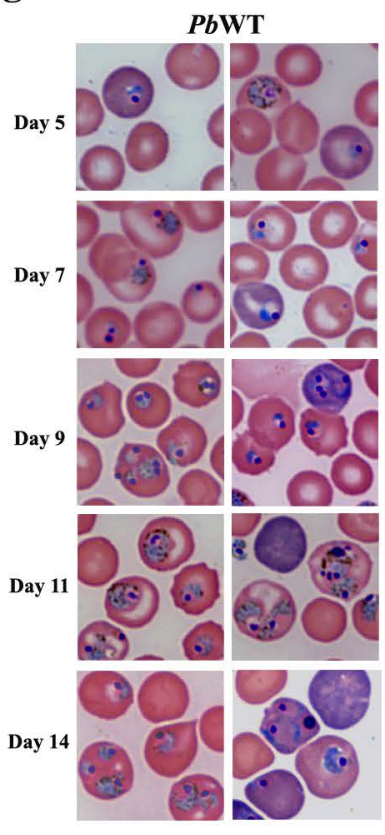

PbALASKO

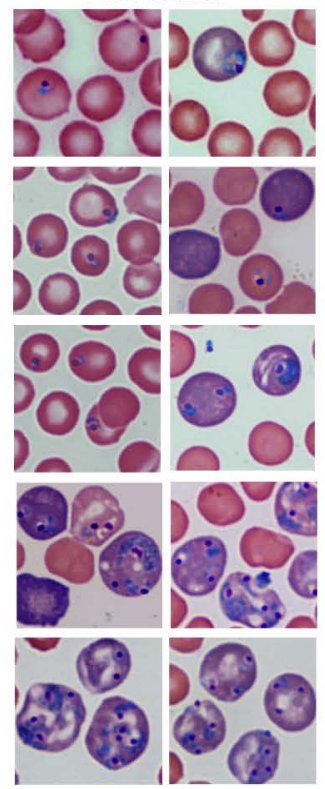

PbFCKO

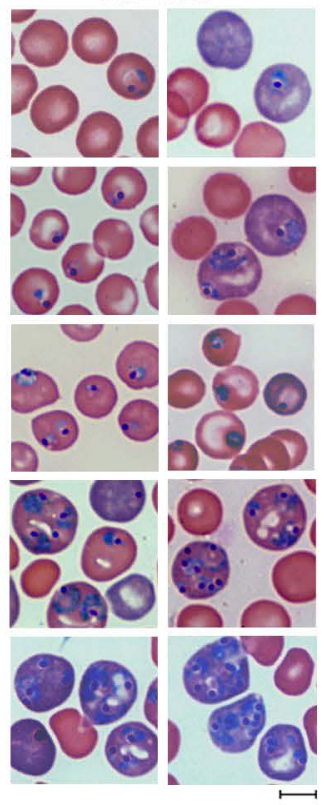

h

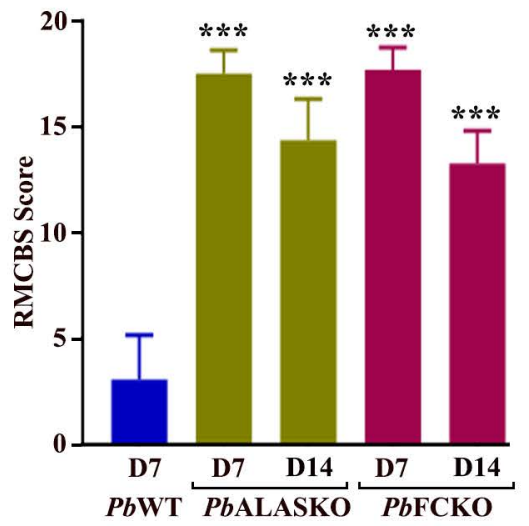

Figure 1 
a

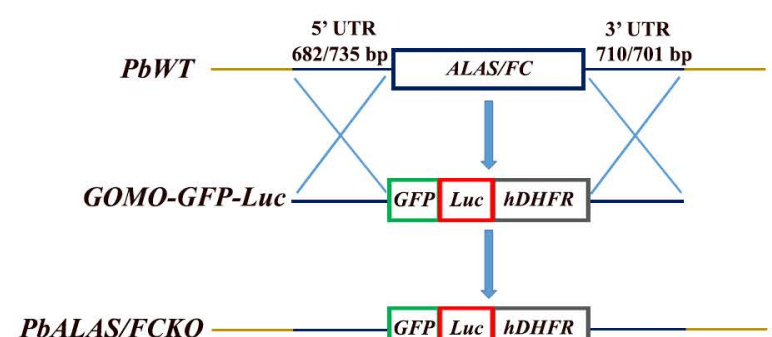

d
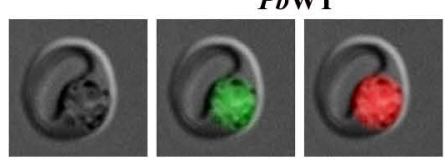

PbALASKO
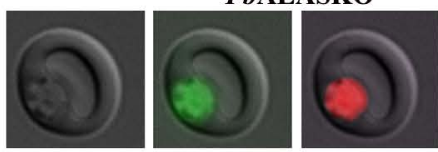

PbFCKO
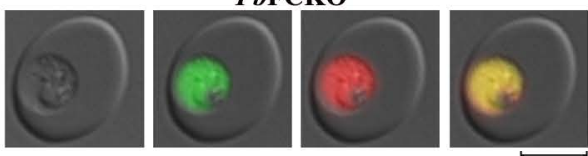

f
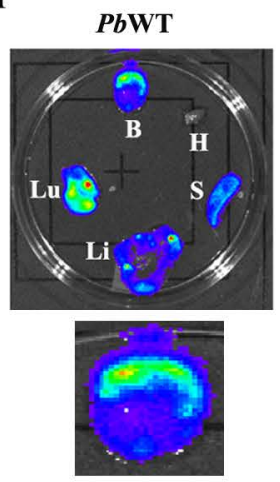

e
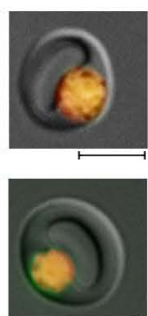

PbALASKO
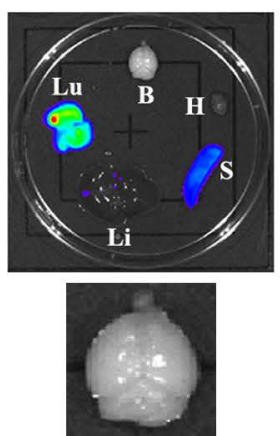

b

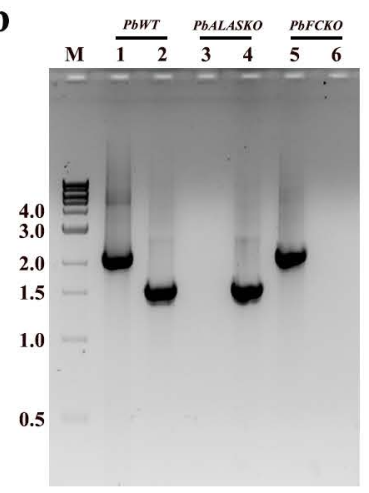

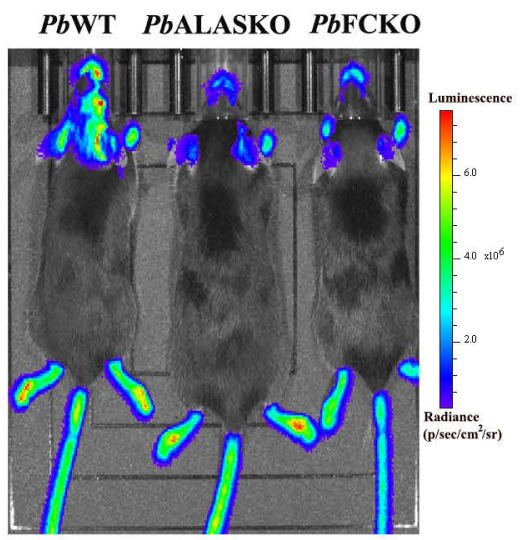

PbWT PbALASKO PbFCKO

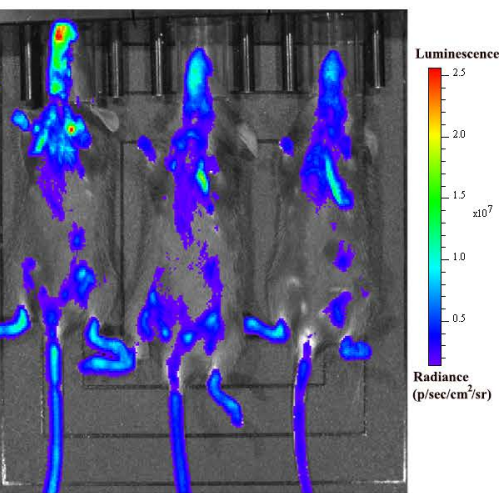

g

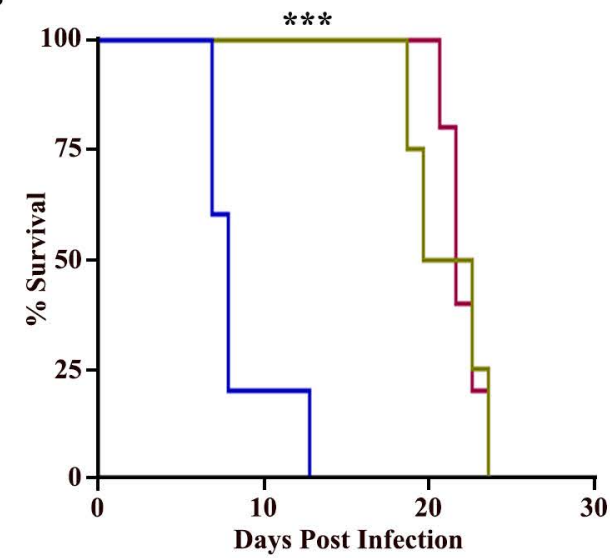

Figure 2 
$\mathbf{a}$

D7

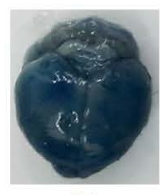

D8

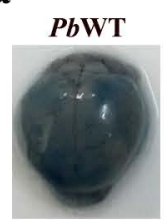

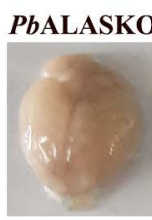

D7

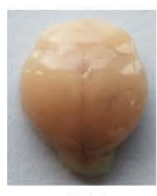

D14

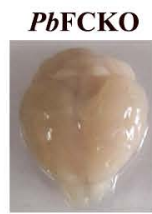

D7

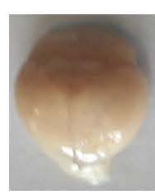

D14

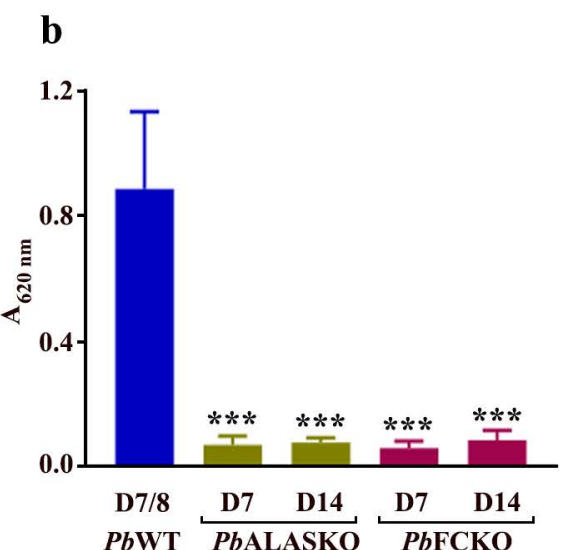

c
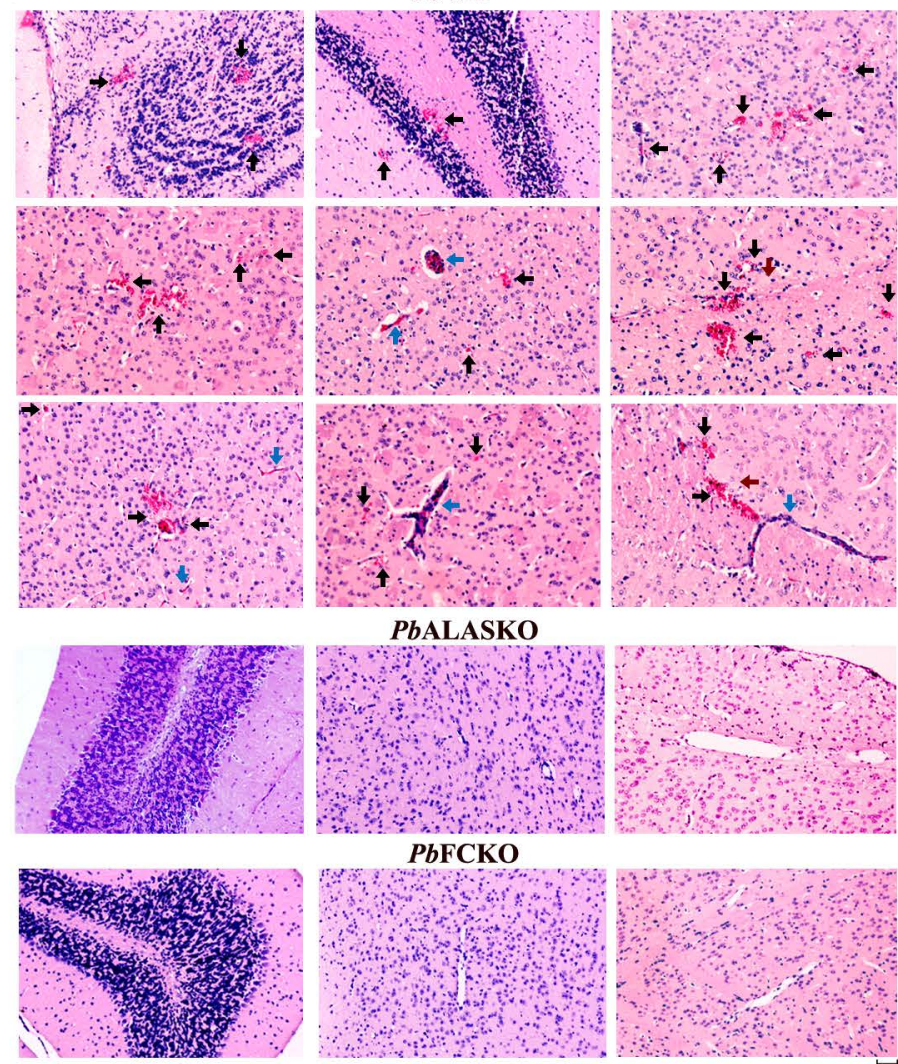
PbFCKO

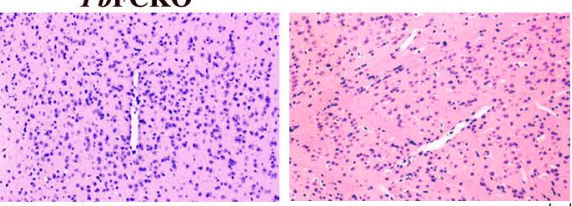

PbWT
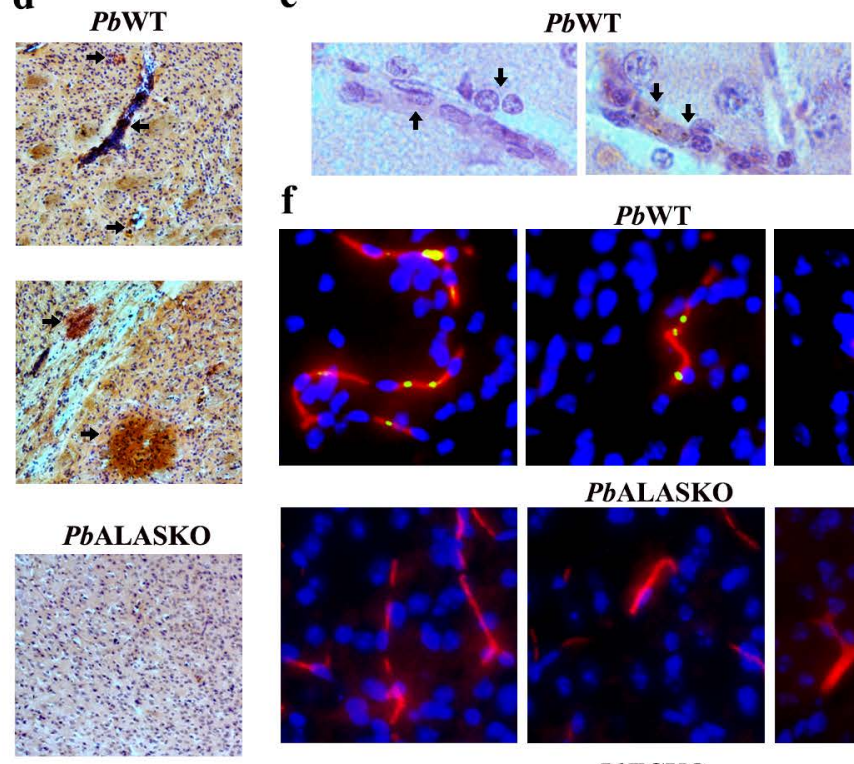

PbALASKO
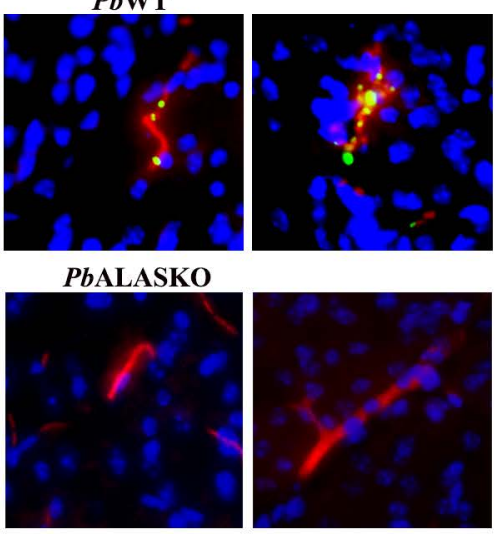

PbFCKO
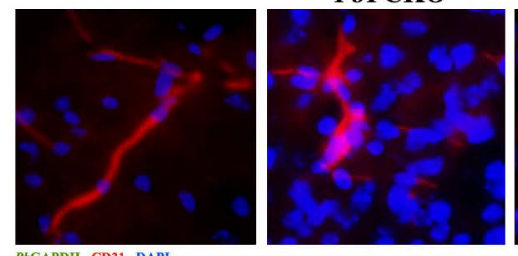

PbALASKO
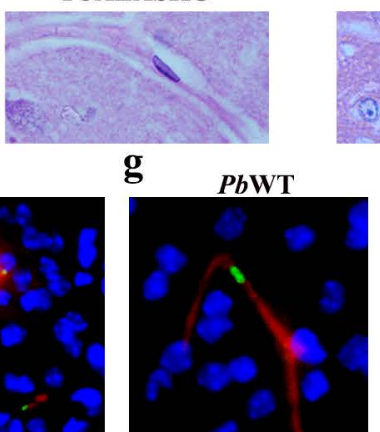

PbALASKO

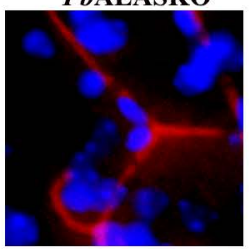

PbFCKO
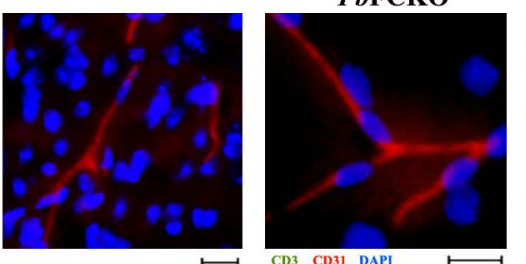

CD3 CD31 DAPI
PbFCKO
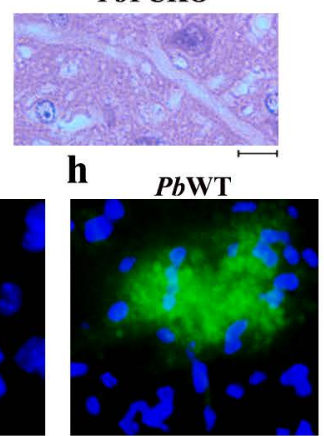

PbALASKO

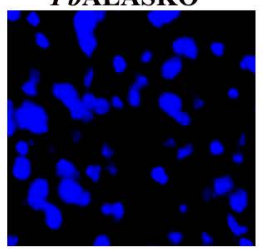

PbFCKO

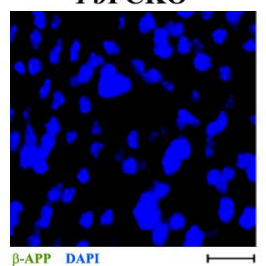

Figure 3 
a
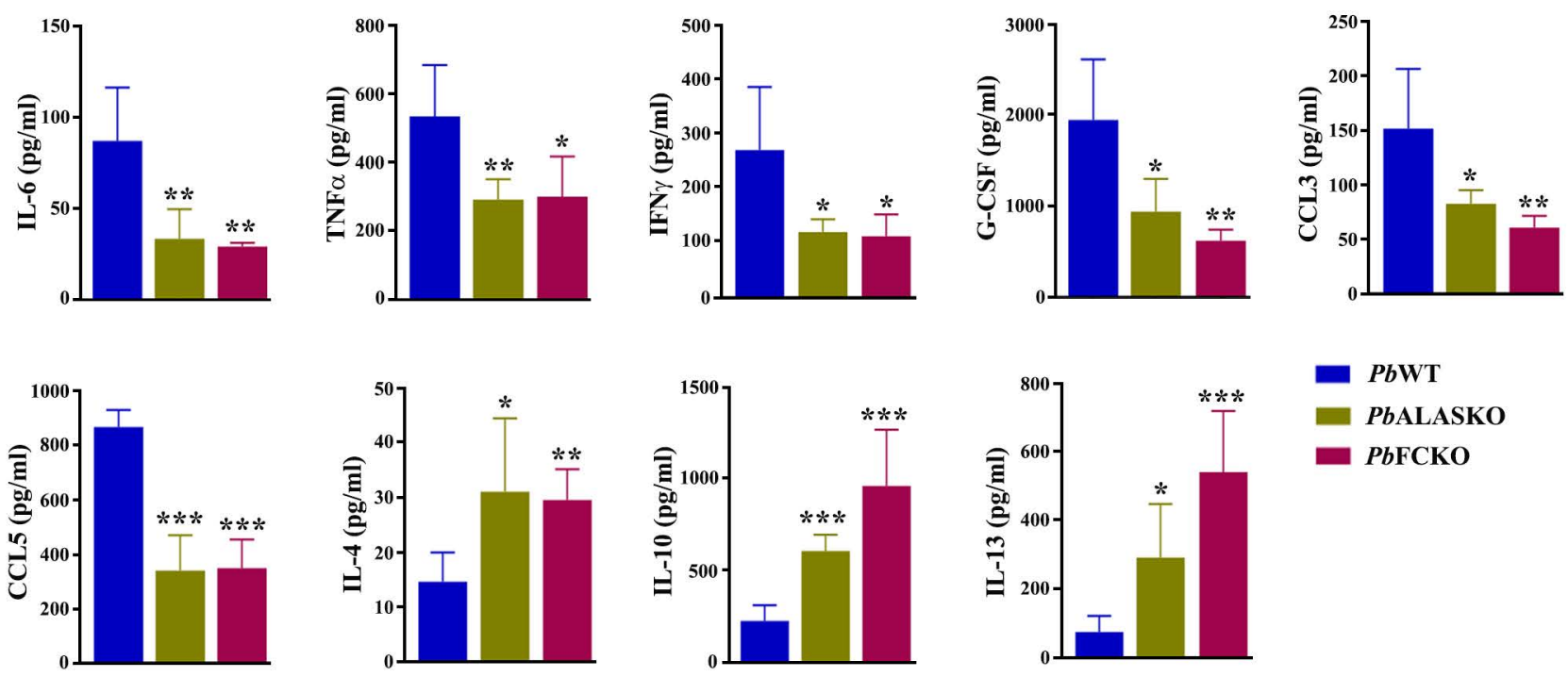

- PbWT

- PbALASKO

- PbFCKO

b

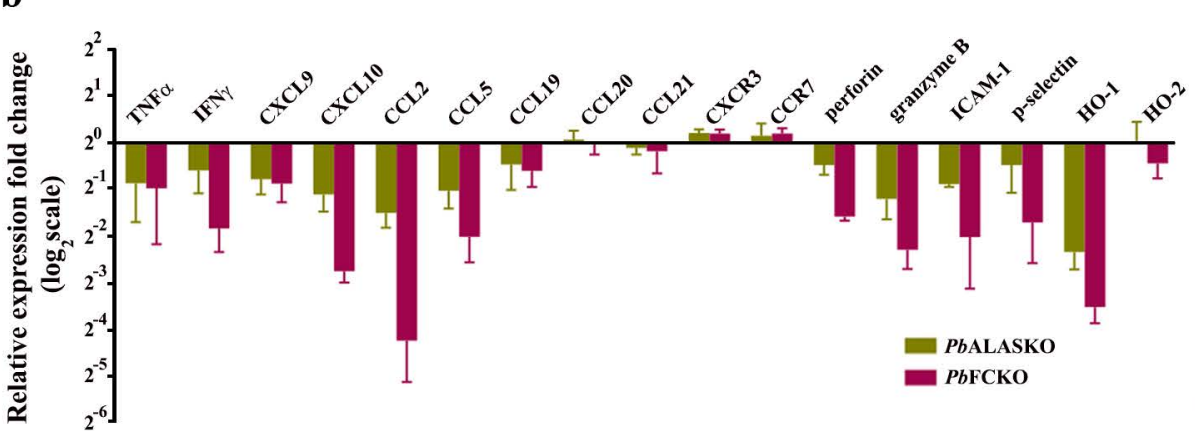

d

c

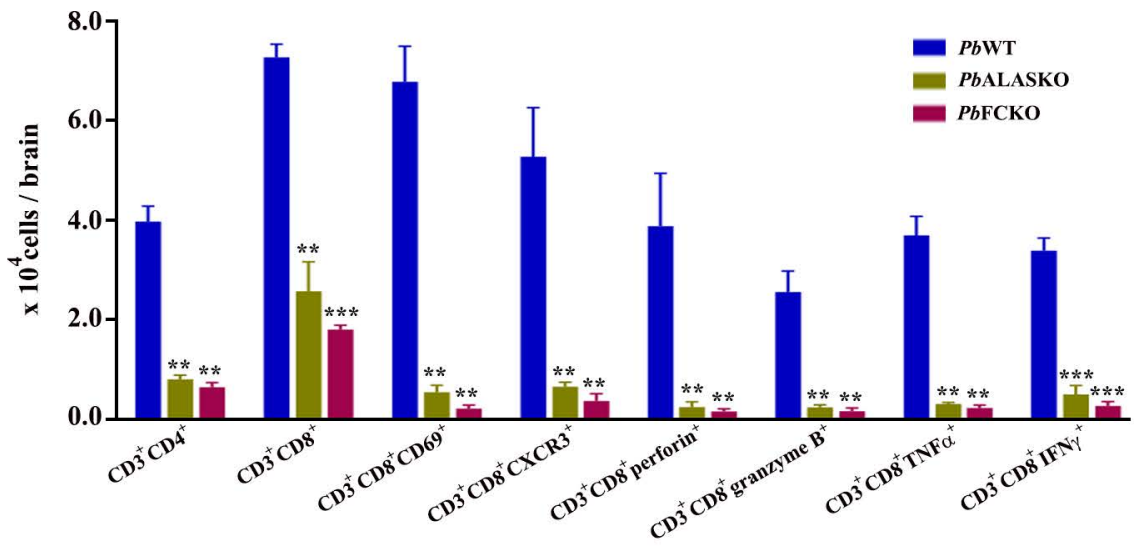

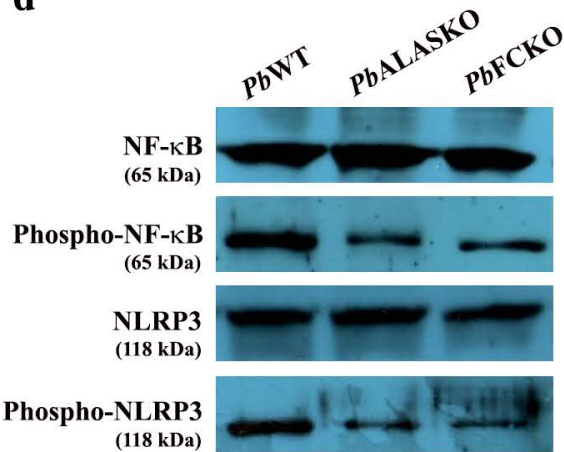

Procaspase-1

(48 kDa)

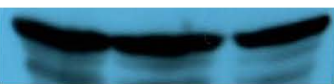

Caspase-1 (22 kDa)

Pro-IL-1 $\beta$ (32 kDa)

IL-1 (17 kDa)

Actin (42 kDa)

Figure 4 
$\mathbf{a}$

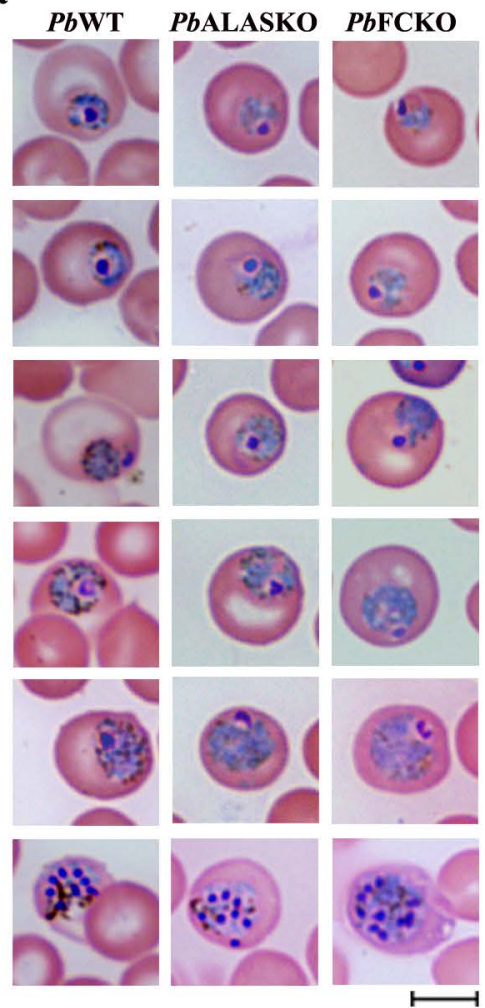

f

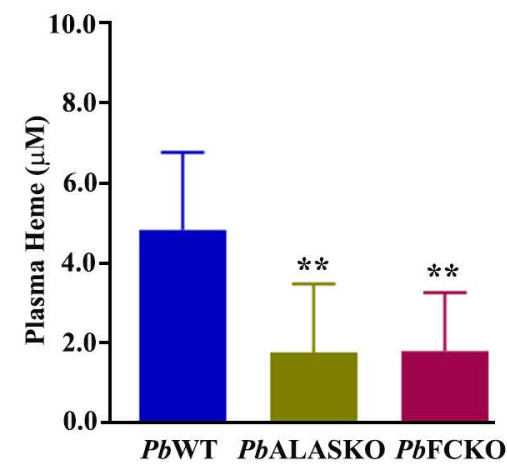

i

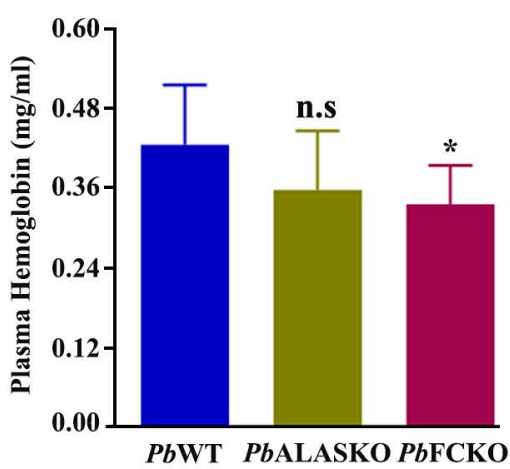

b c

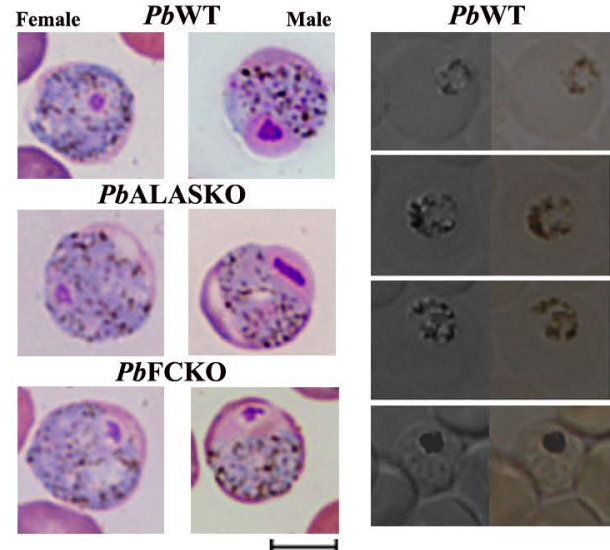

d

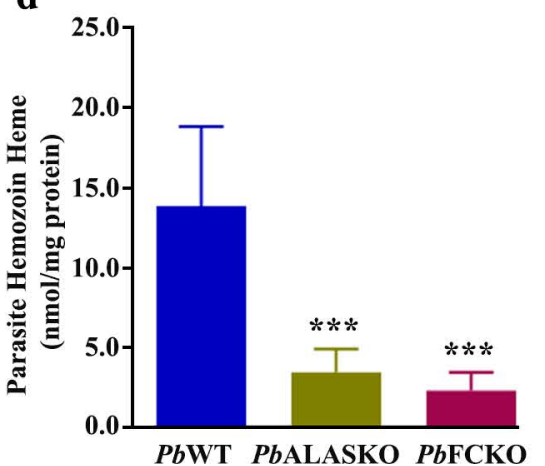

g
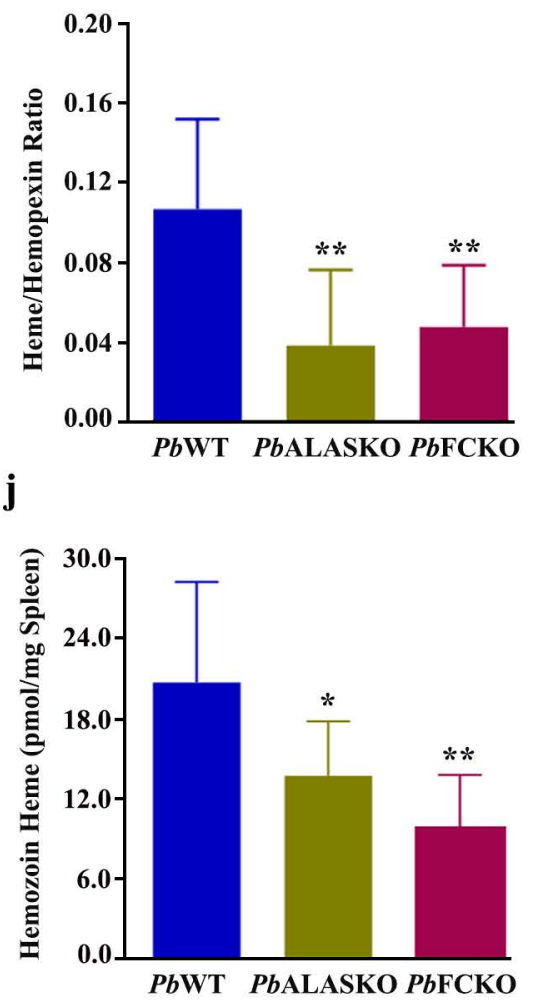

Figure 5
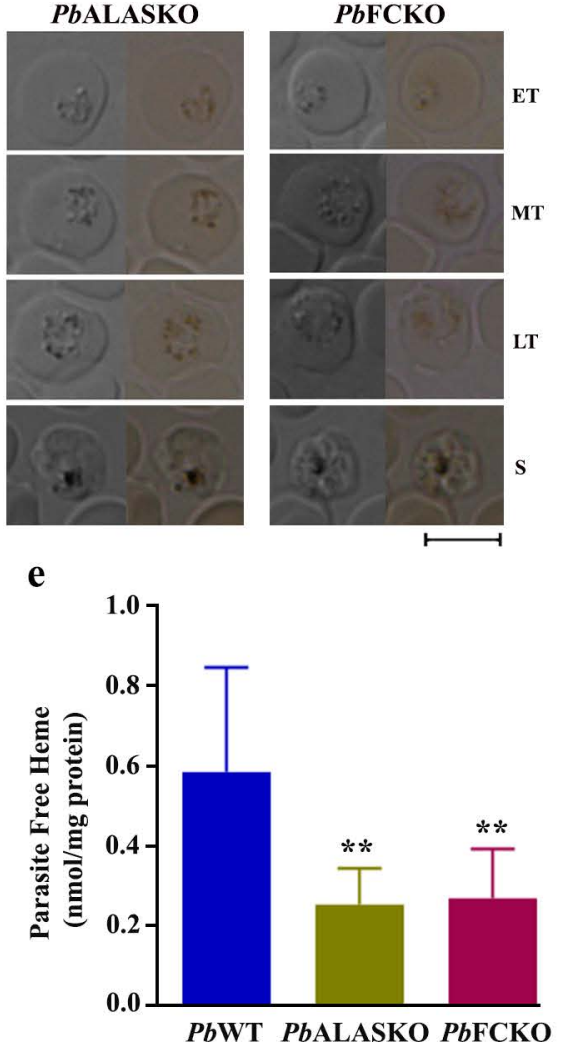

h

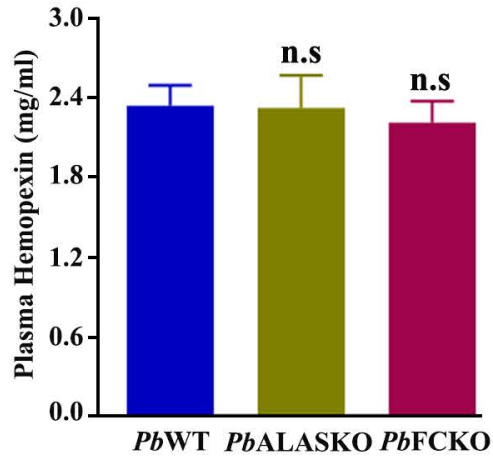

k

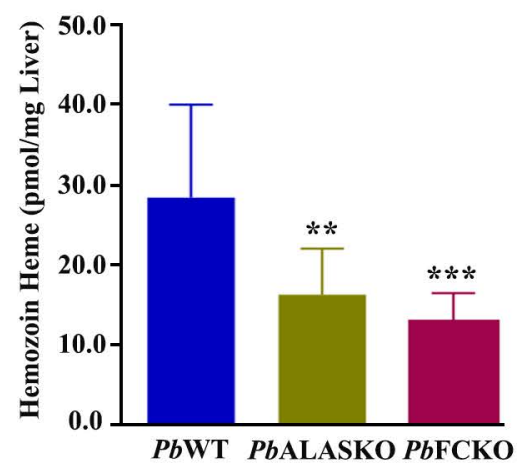



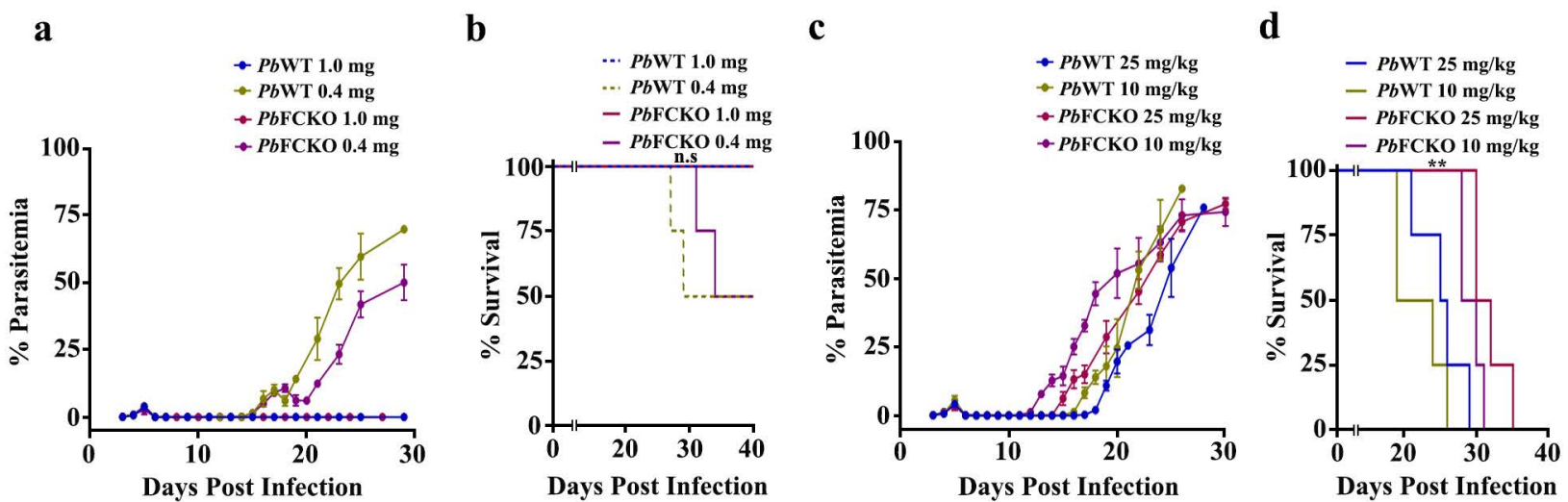

Figure 6 
a

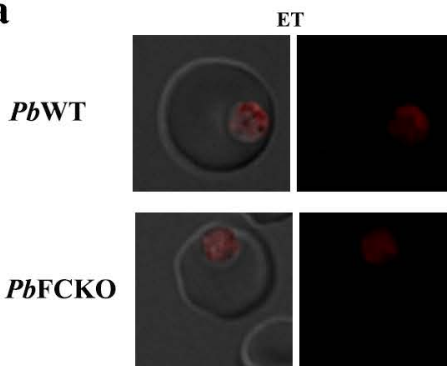

b

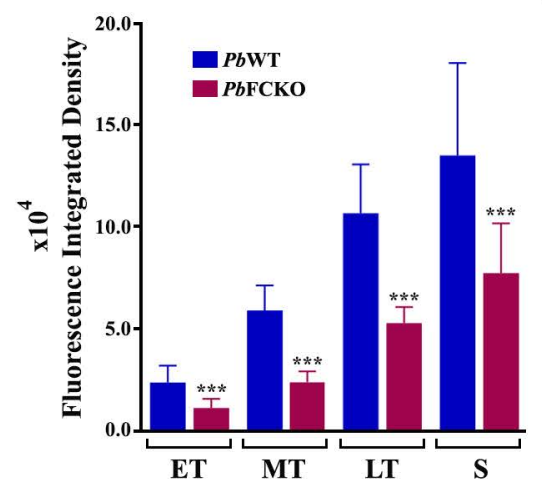

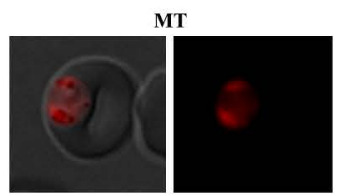

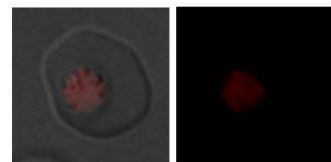

c

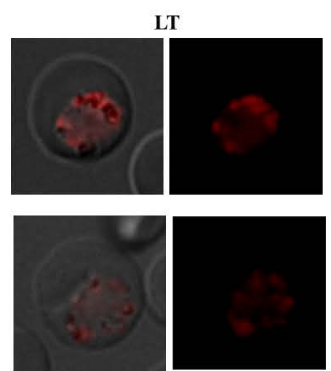

d
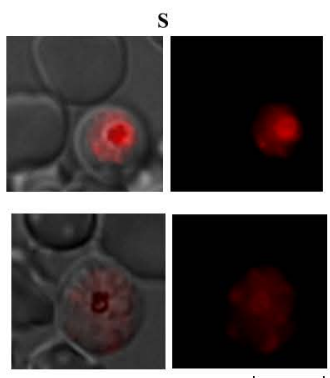

d

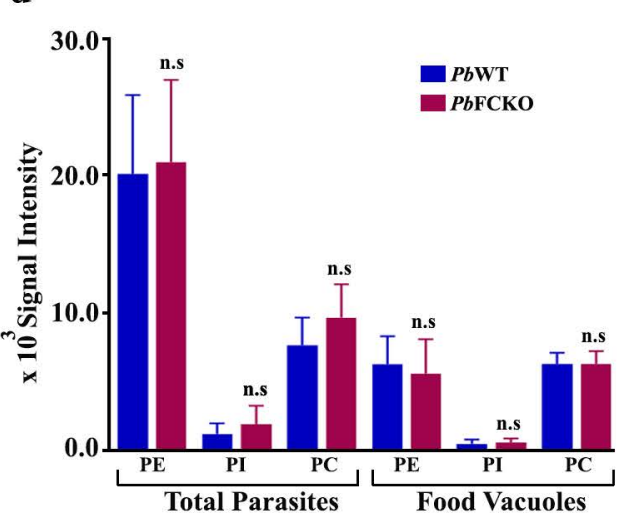

e

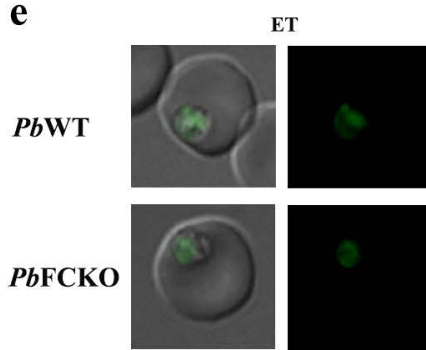

f

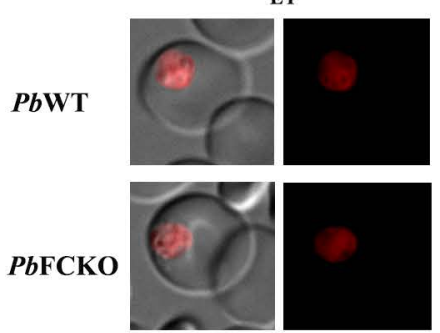

Total Parasites Food Vacuoles PbWT PbFCKO PbWT PbFCKO

PbWT PbFCKO PbWT PbFCKO
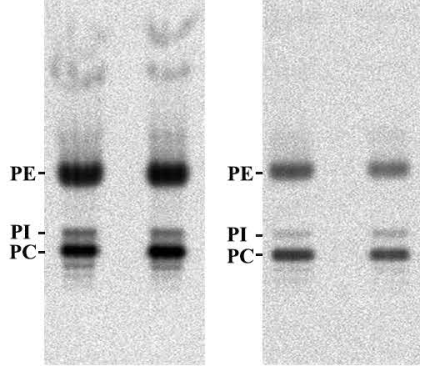

MT
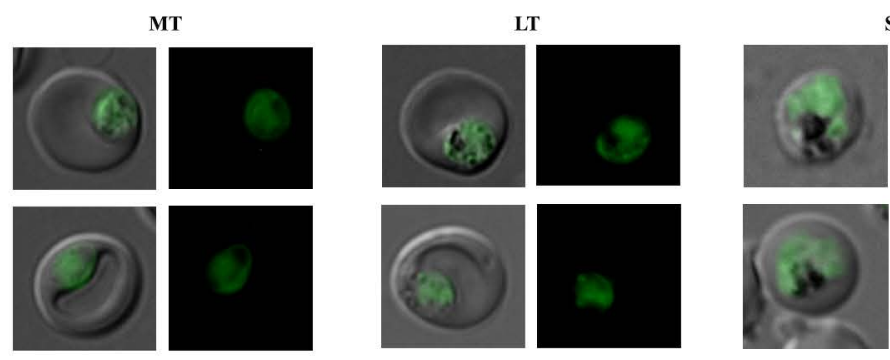

MT

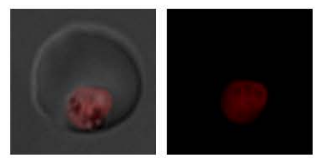

LT
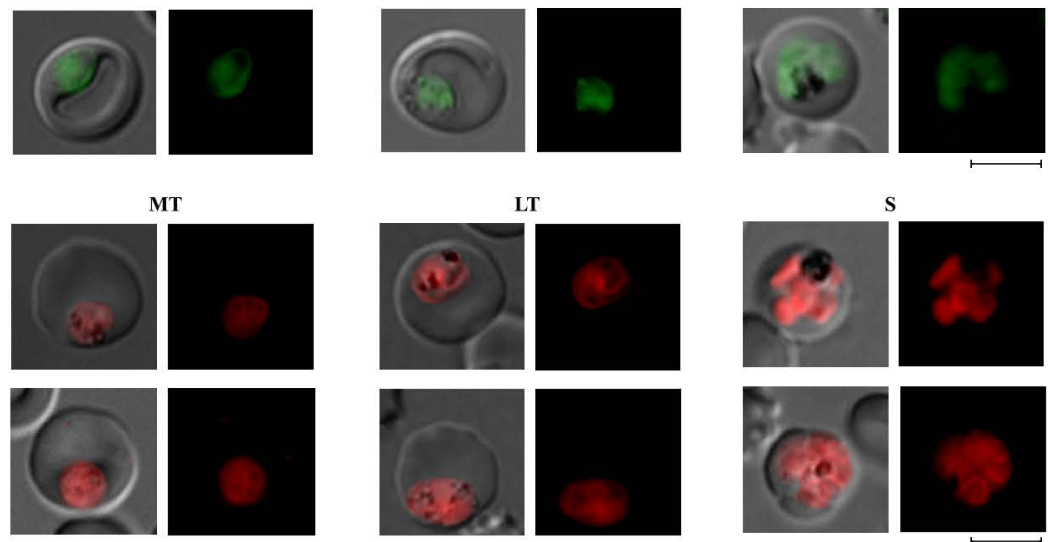

g

h
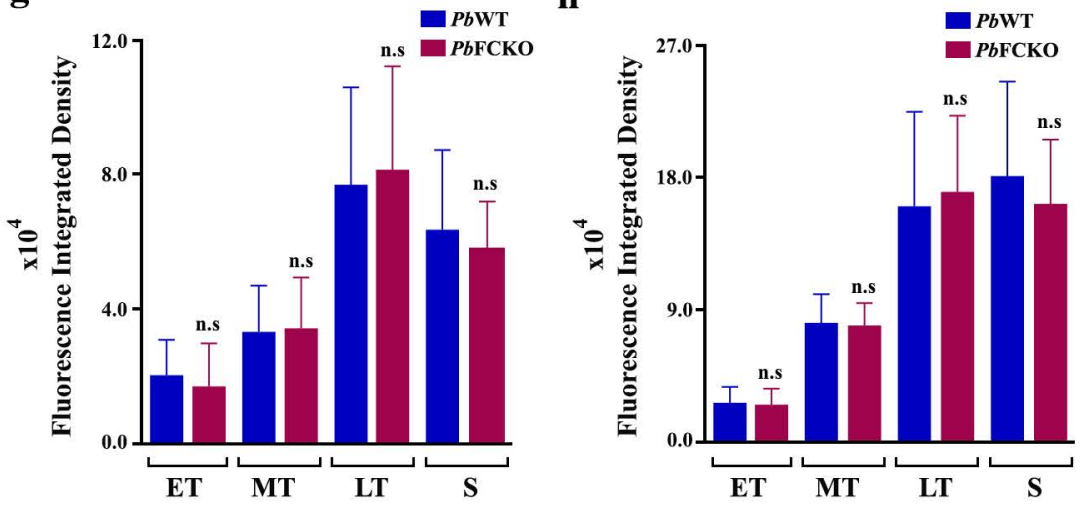

Figure 7 
a

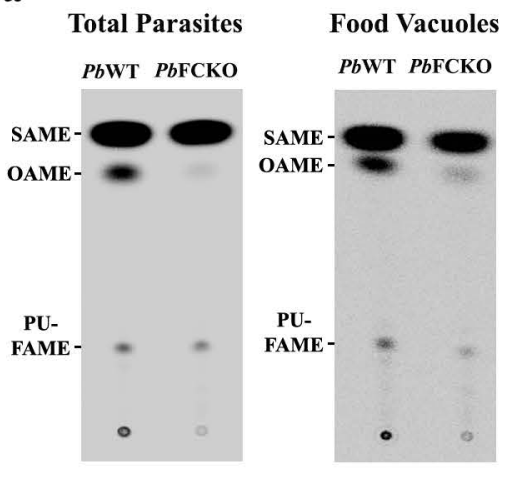

d

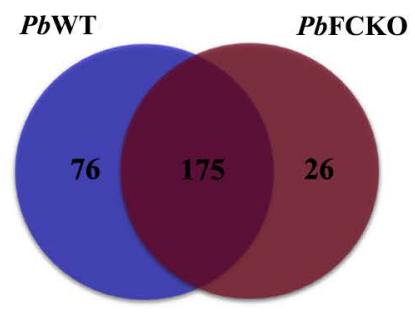

b

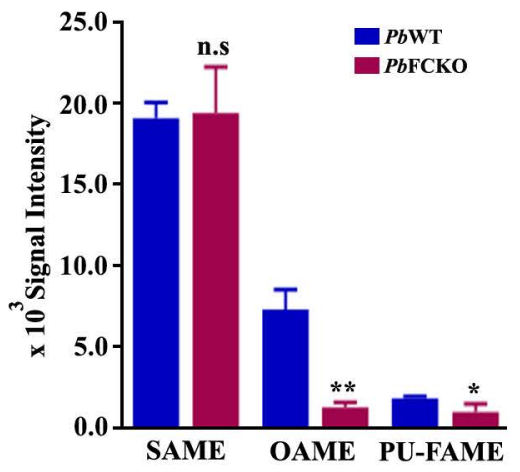

e
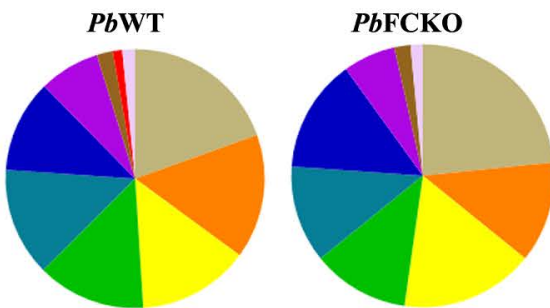

$\square$ Nucleic acid and protein synthesis $\square$ Host-trafficked and parasite membraneassociated proteins

$\square$ Cytoskeleton and organelle function Chaperones

Metabolic enzymes

Histones and nucleic acid binding proteins

$\square$ Proteasome-associated proteins

$\square$ Hemoglobin degradation

$\square$ pH regulation

$\square$ Unknown function

Figure 8 
a

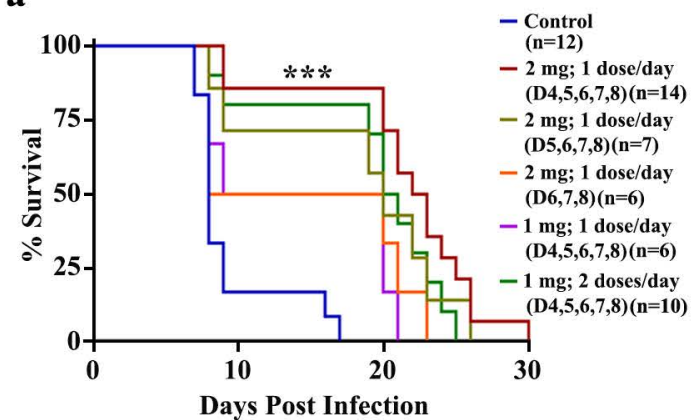

d
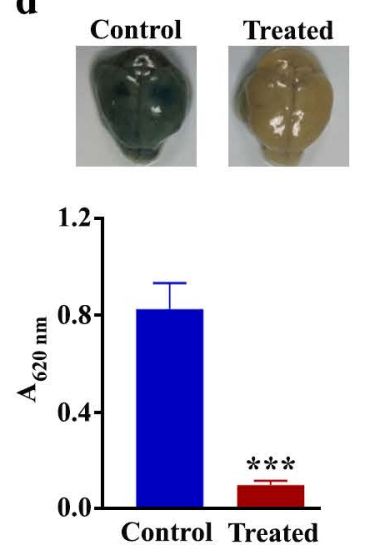

e

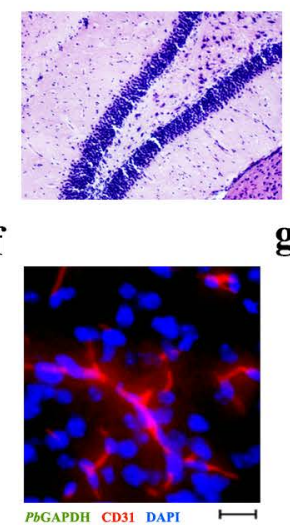

b

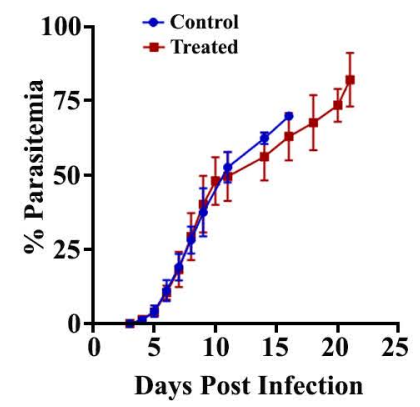

c
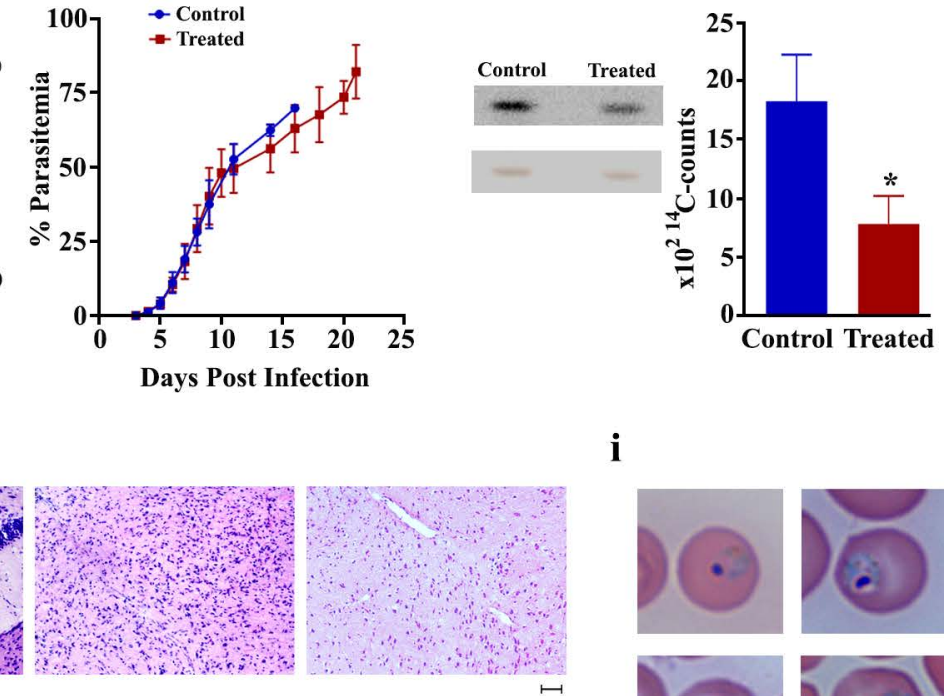

h
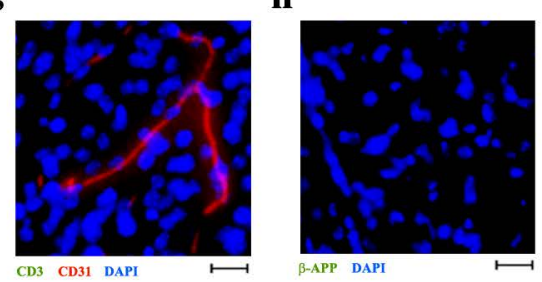

i
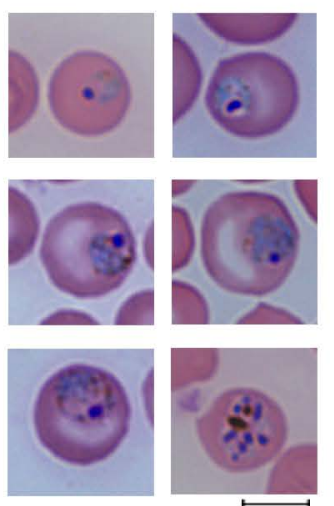

n

0

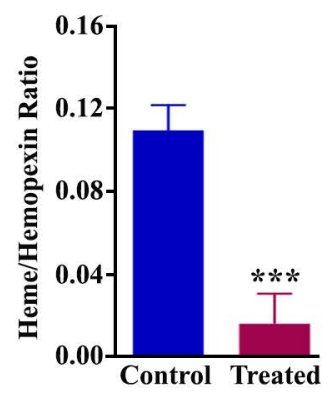

k

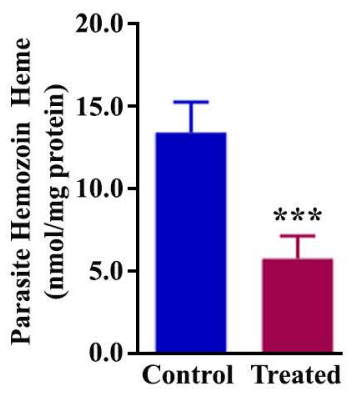

p

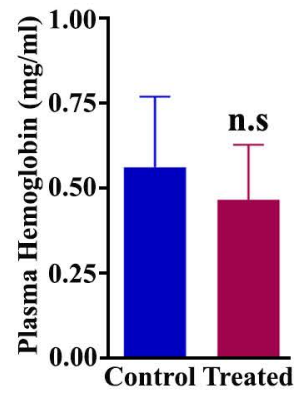

m

I
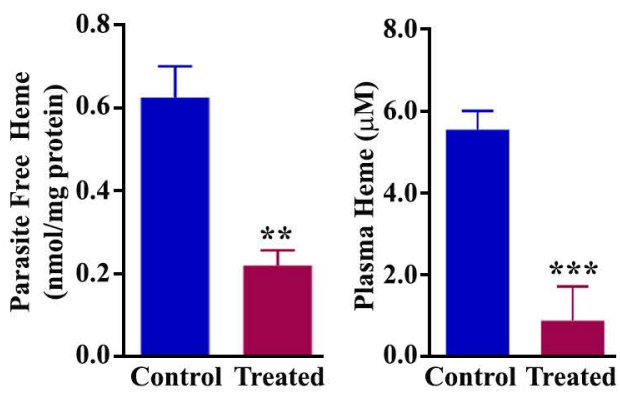

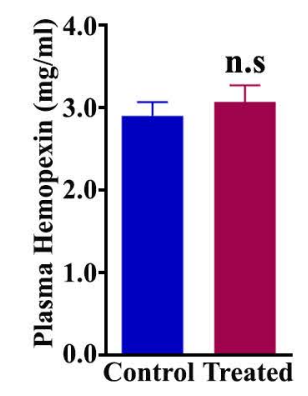

Figure 9 


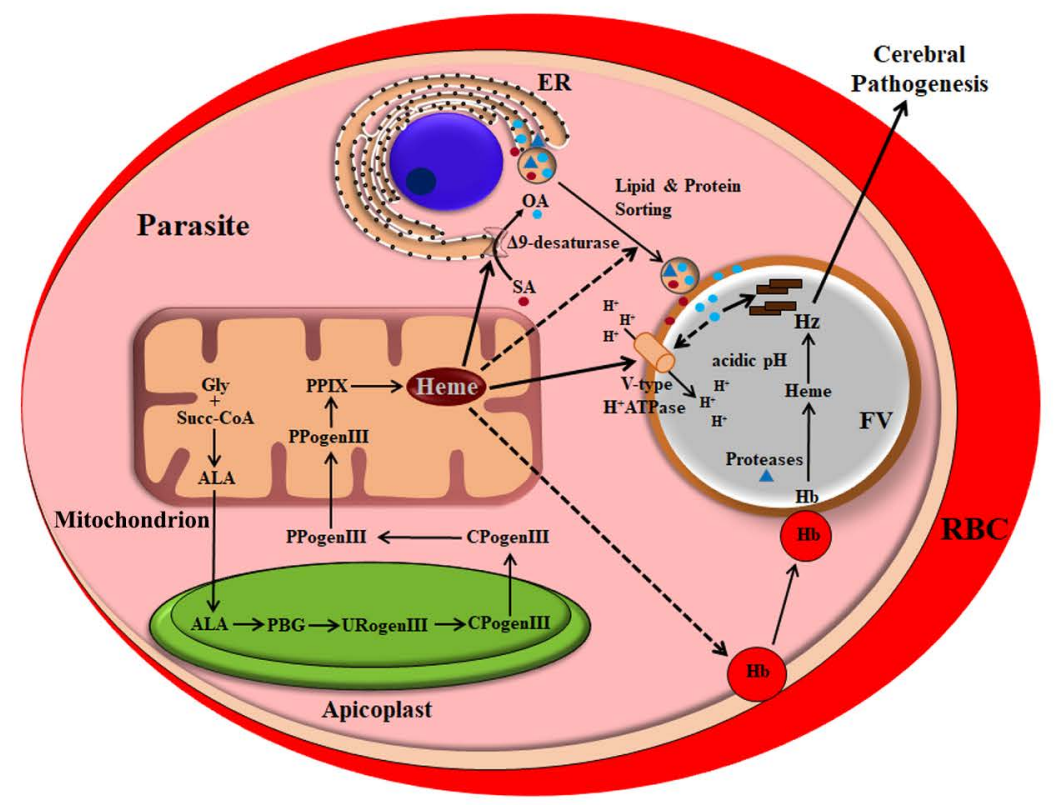

Figure 10 
a

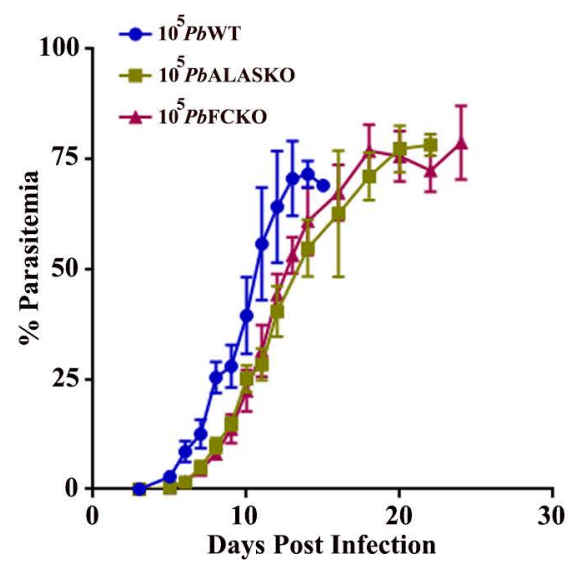

b

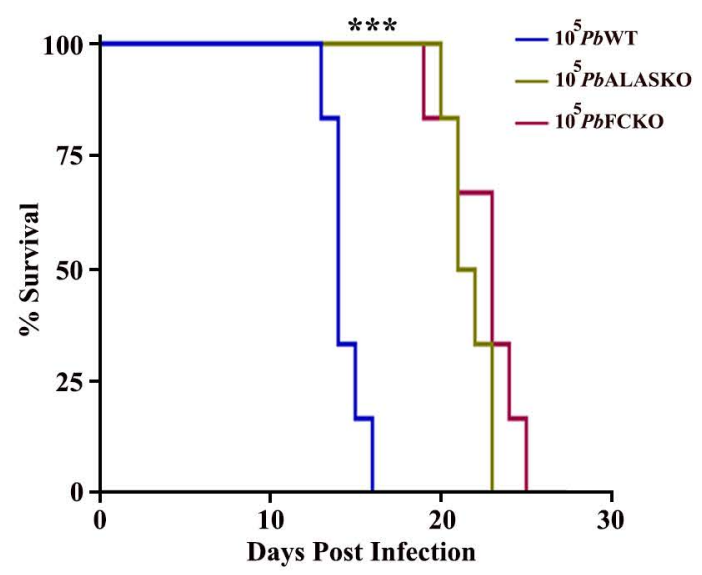

Figure S1 


\section{PbWT}
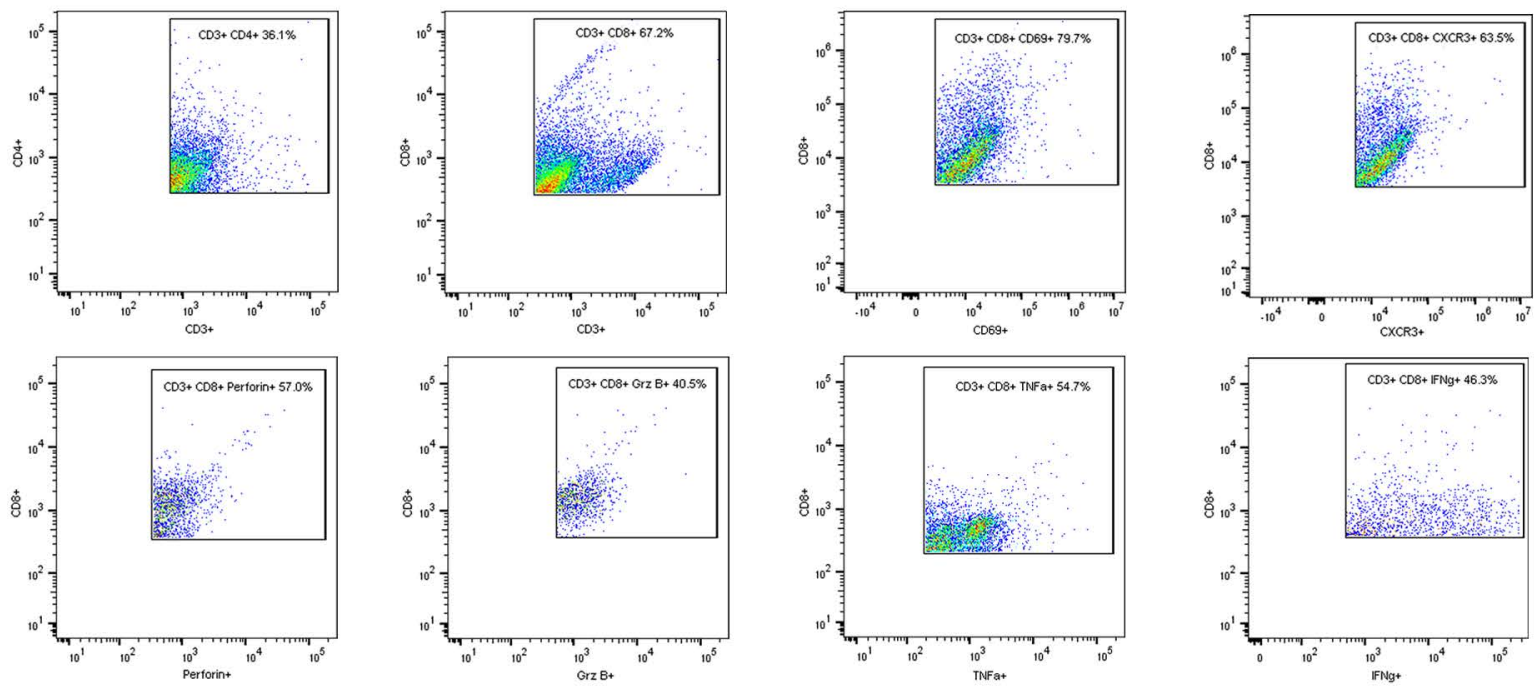

PbALASKO
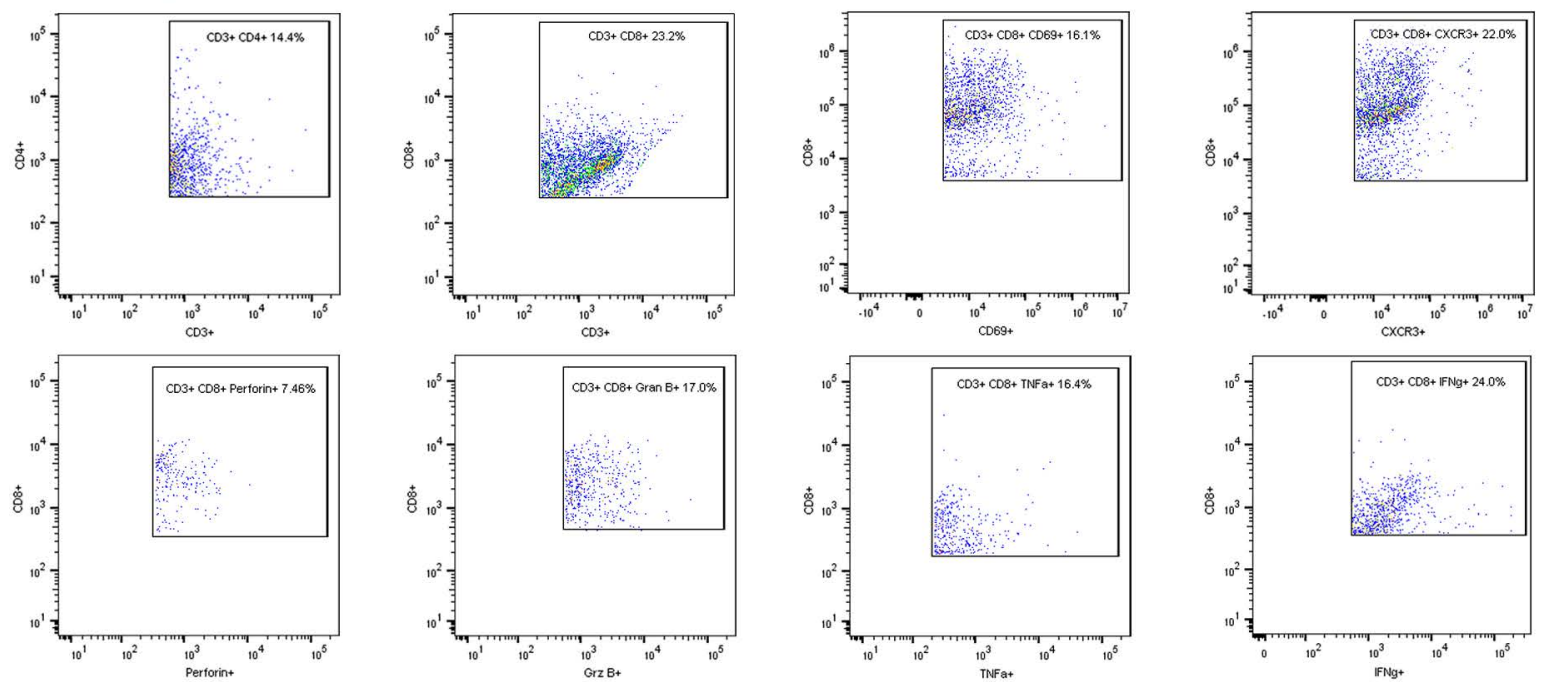

\section{PbFCKO}
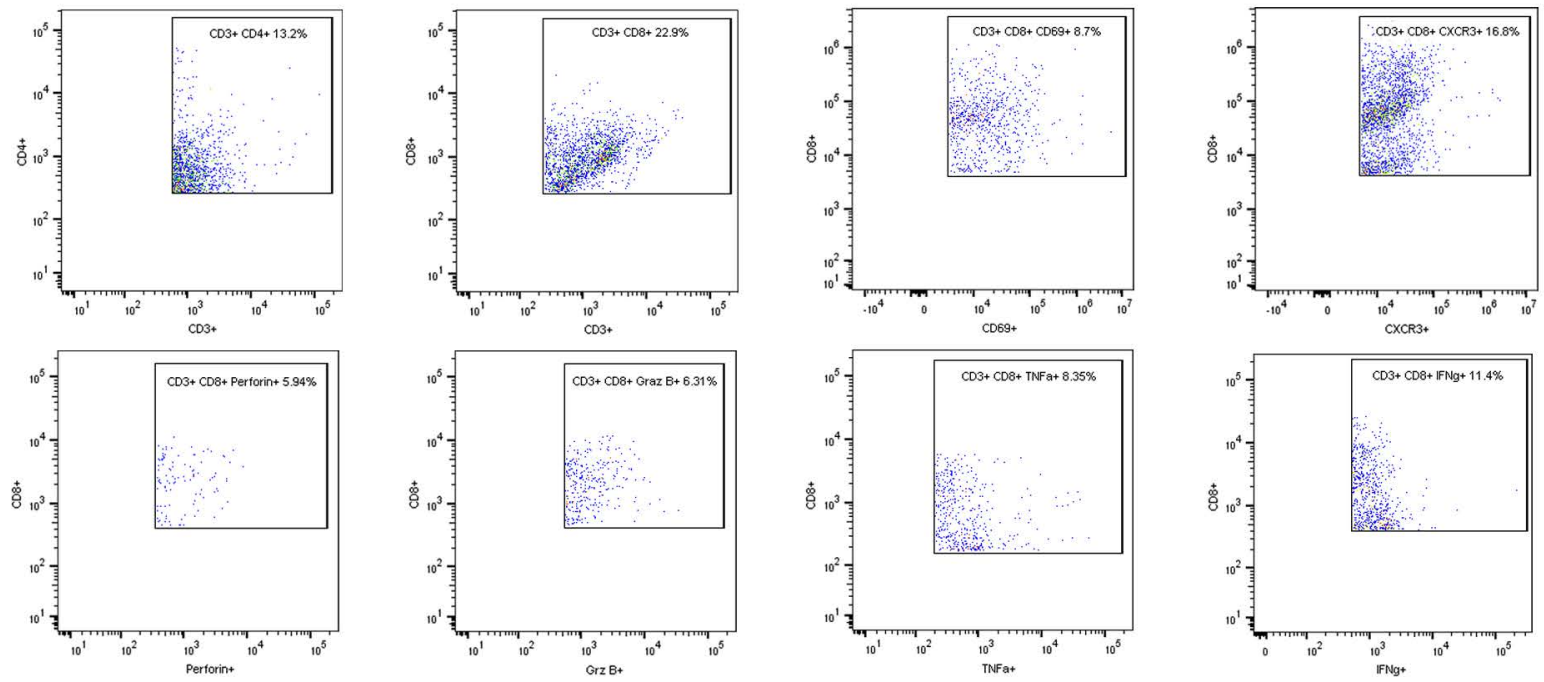

Figure S2 
a

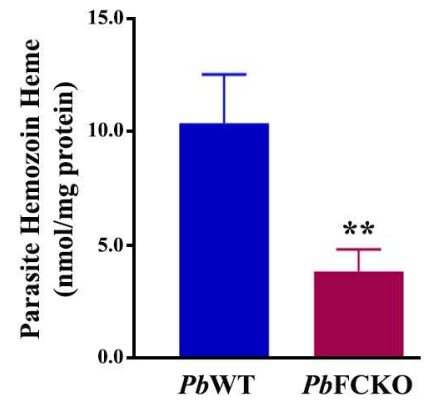

b

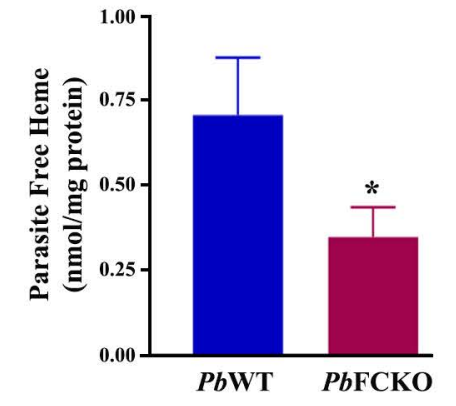

Figure S3 
1,2 - PbWT

3,4 - PbFCKO

a

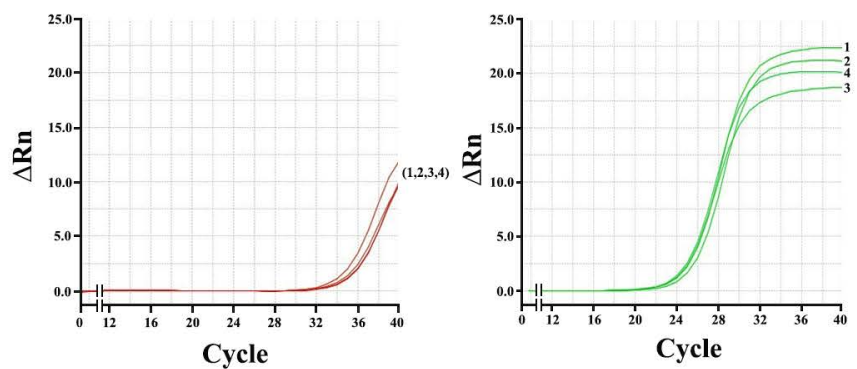

e

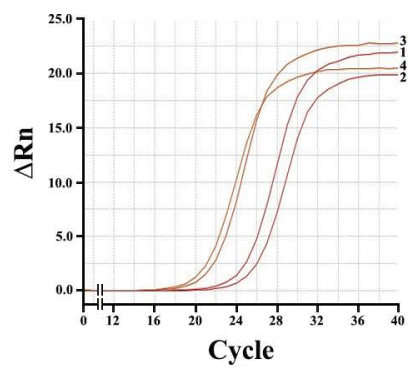

b c

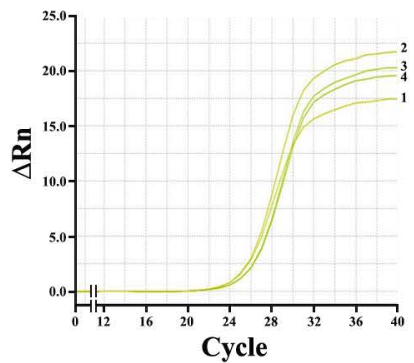

g

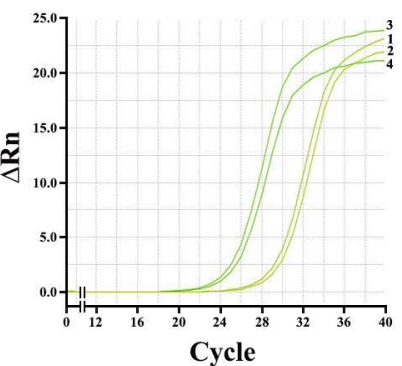

d

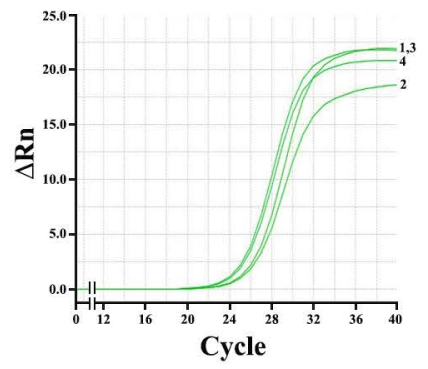

1,2 - PbWT

3,4 - PbFCKO

h

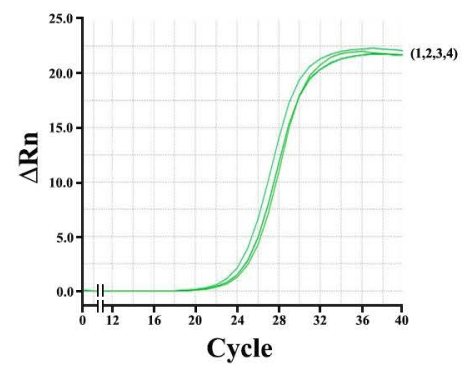

Figure S4 


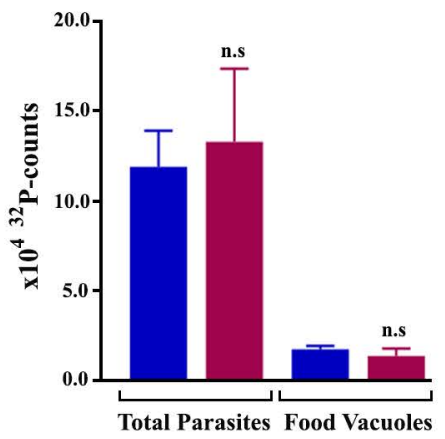

Figure S5 
Oleic acid methyl ester

Compound Information

Peak Name:

9-Octadecenoic acid (Z)-, methyl ester
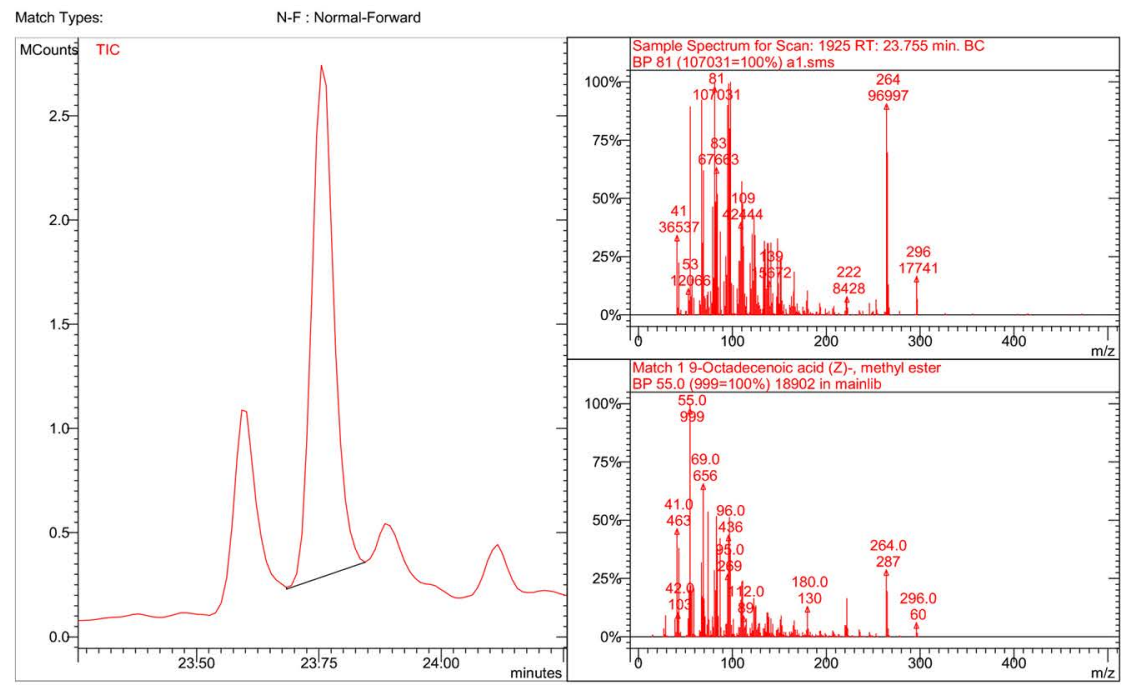

Arachidonic acid methyl ester

Compound Information

Peak Name: $\quad 5,8,11,14$-Eicosatetraenoic acid, methyl ester
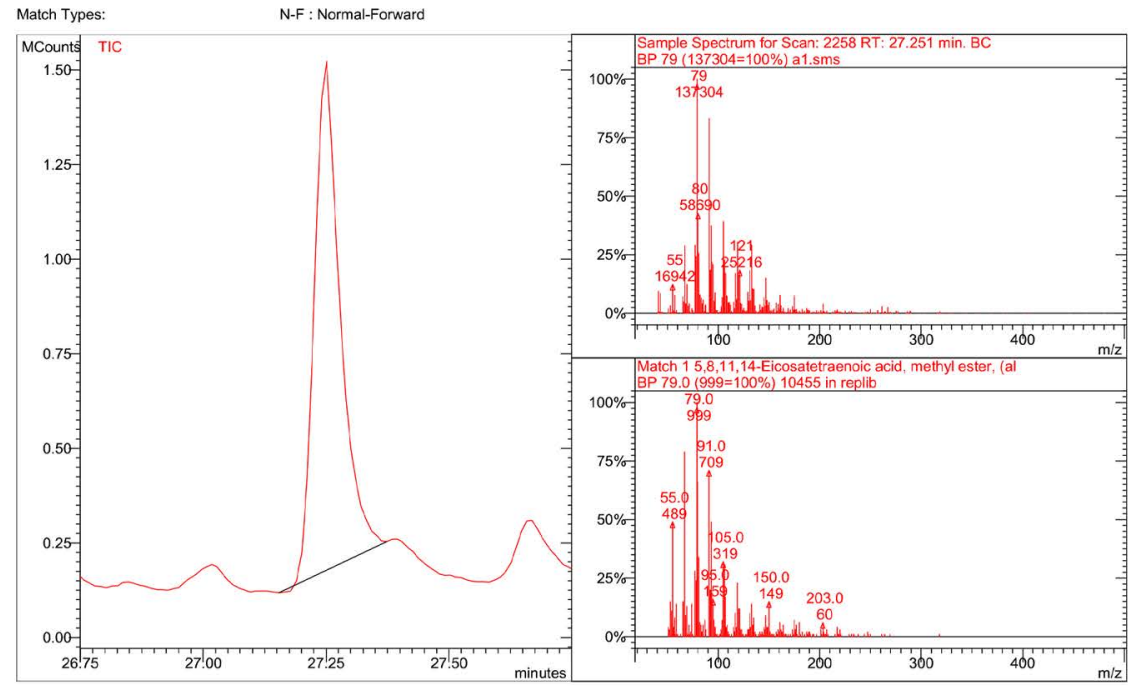

Figure S6 b

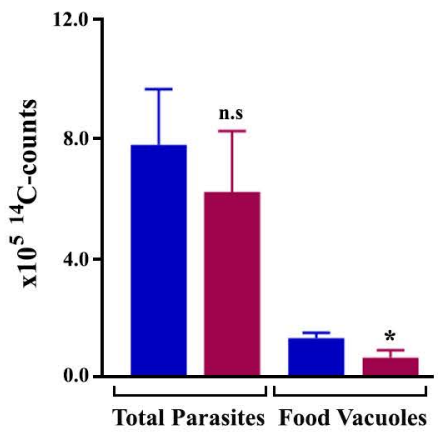

